\title{
INVERTED FINITE ELEMENTS: A NEW METHOD FOR SOLVING ELLIPTIC PROBLEMS IN UNBOUNDED DOMAINS
}

\author{
TAhar Zamène BoulmeZaOud ${ }^{1,2}$
}

\begin{abstract}
In this paper, we propose a new numerical method for solving elliptic equations in unbounded regions of $\mathbb{R}^{n}$. The method is based on the mapping of a part of the domain into a bounded region. An appropriate family of weighted spaces is used for describing the growth or the decay of functions at large distances. After exposing the main ideas of the method, we analyse carefully its convergence. Some 3D computational results are displayed to demonstrate its efficiency and its high performance.
\end{abstract}

Mathematics Subject Classification. 35J, 35J05, 65Jxx, 65Nxx, 65Rxx.

Received: December 15, 2003.

\section{INTRODUCTION}

A wide class of problems encountered in physics and engineering are originally formulated in a media with an infinite extent. Besides electromagnetic problems, these problems concern computational fluid dynamics, acoustic, geophysics, elasticity, magnetohydrodynamics, astrophysics and several other fields of research. Consequently, the conception of efficient numerical methods which are suitable for unbounded domains is of crucial importance for the solving and the understanding of such problems. Reviewing the available strategies for solving PDEs in unbounded domains, we find a variety of methods having various degrees of accuracy, flexibility and sophistication. However, most of the existing methods rely

- either on an integral representation of the exact solution and the use of Boundary Elements (see, e.g., $[15,22,23,32,34-36])$;

- or on replacing the unbounded domain by a sufficiently large bounded domain enclosed by a Perfectly Matched Layer (PML) (see $[5,6]$ ), or on the boundary of which an artificial boundary condition is prescribed;

- or on a polar expansion of the solution like in spectral methods (see, e.g., $[12,24])$ or in infinite elements methods (see, e.g., $[7,11,16,18,19,28]$ ).

Surprisingly, little attention was given to methods which consist in mapping the unbounded domain into a bounded one. This seems to be due to the appearance of a singularity after mapping.

Keywords and phrases. Unbounded domains, inverted elements method, weighted Sobolev spaces.

${ }^{1}$ Laboratoire de Mathématiques, Université de Versailles Saint-Quentin en Yvelines (UVSQ), 45 avenue des États-Unis, Bâtiment Fermat, 78035 Versailles, France.

${ }^{2}$ Laboratoire Jacques-Louis Lions, Université Paris VI, BC187, 75252 Paris Cedex, France. boulmeza@math.uvsq.fr 
Our aim in this paper is to propose a new method which we call Inverted Finite Element Method (IFEM) for solving elliptic PDEs in infinite domains. The IFE method relies on the partition of the infinite domain into a bounded sub-domain, in which usual finite elements are used, and an unbounded sub-domain which is mapped into a bounded region by means of polygonal inversions. The method is of an arbitrary degree and is exactly conforming. The use of an adequate family of weighted Sobolev spaces for describing the behavior of functions at large distances is at the heart of our approach. The other ideas of the method are close to those of the finite element method. Among the advantages of the IFEM, let us underline the absence of artificial boundaries and artificial conditions. In addition, the method offers the possibility of truncating the computational domain with polygonal or box-like boundaries. On the other hand, there is no need to know a priori the precise behavior at infinity of the solution, but only some simple integrability conditions. In practice, the method leads to linear systems with sparse matrices and whose size and shape are similar to finite elements matrices.

For the sake of simplicity, the Inverted Finite Element Method will be applied here to linear elliptic problems which admit an abstract variational formulation as follows:

Find $u \in W$ such that

$$
\forall v \in W, a(u, v)=\ell(v)
$$

where $W$ denotes a weighted Sobolev space whose elements are functions defined on an unbounded region, $a(.,$.$) is a bilinear form and \ell($.$) a linear form. In Section 2, we show how some usual second and fourth order$ problems in unbounded domains (e.g., the whole space, the half-space, exterior regions, etc.) can be written into form (1), with $a(.,$.$) and \ell($.$) satisfying usual well-posedness conditions. In that case, the space W$ is often an adequate weighted Sobolev space whose weights are of the form $(1+|\boldsymbol{x}|)^{\alpha}, \alpha \in \mathbb{R}$. Indeed, this kind of weighted spaces turned out to be natural for solving successfully elliptic problems and for describing their solutions in unbounded domains (see $[3,4,8-10,22,25,29]$ ). This is due to multiple reasons. The first reason is the possibility of describing and classifying easily the decay or growth of functions at infinity. Another reason lies in the fact that they allow to retrieve most of the fine functional properties of Sobolev spaces in bounded domains, and which are in general lost when the domain is unbounded: Poincaré's inequality, Green's formula, compact imbeddings (see, e.g., $[1,13]$ ), etc.

This paper is organized as follows:

- In Section 2, we review the definitions and the basic properties of a family of weighted spaces in unbounded domains. Some weighted spaces in bounded regions containing a singularity are also presented. The treatment of some typical elliptic problems in unbounded domains is displayed throughout several examples.

- Section 3 contains a presentation of the Inverted Element Method. The notions of polygonal inversion and polygonal Kelvin transform are particularly detailed.

- In Section 4, we deal with the analysis of the best approximation error. Firstly, we introduce an interpolation operator corresponding to the mesh of the domain. Then, we give an estimate of the local and the global interpolation errors.

- In Section 5, we give an estimate of the error of approximation when the method is applied to a model elliptic problem. We prove in particular that the rate of convergence is the same as the rate of convergence of Finite elements in a bounded domain.

- In Section 6, we give a general outline of the implementation of the method in the case of the 3D Laplace equation in the half-space. The computation of the stiffness matrix is treated in detail. The performance of the method, the validity of the error estimates and the influence of various parameters are investigated throughout some computational tests.

In the sequel, the notation $a \lesssim b$ (resp. $a \simeq b$ ) means that there exists a constant $c$ (resp. two constants $c_{1}$ and $c_{2}$ ) independent of the discretization parameter $h$ and the involved functions such that $a \leq c b$ (resp. $\left.c_{1} b \leq a \leq c_{2} b\right)$ 


\section{Weighted Sobolev spaces}

In all the paper $n \geq 1$ denotes a non-negative integer. For any multi-index $\mu=\left(\mu_{1}, \ldots, \mu_{n}\right) \in \mathbb{N}^{n}$ we set $|\mu|=\mu_{1}+\ldots+\mu_{n}$. For any real number $r>0, B_{r}$ (resp. $S_{r}$ ) is the open ball (resp. the sphere) of $\mathbb{R}^{n}$ of radius $r$ and centered at the origin. Given an integer $k \geq 0, \mathbb{P}_{k}$ denotes the set of all the polynomials in variables $x_{1}, \ldots, x_{n}$ of degree $\leq k . \stackrel{\circ}{\mathbb{P}}_{k}$ is the subspace of $\mathbb{P}_{k}$ formed by all the polynomials $p \in \mathbb{P}_{k}$ such that $p(\mathbf{0})=0$.

Let $\Omega$ be an open set of $\mathbb{R}^{n}$. We denote by $\mathscr{D}(\Omega)$ the space of all $\mathcal{C}^{\infty}$ functions with a compact support included in $\Omega$ and by $\mathscr{D}^{\prime}(\Omega)$ its dual space (the space of distributions). Given an integer $m \geq 0$ and a real number $p \in\left[1,+\infty\left[, W^{m, p}(\Omega)\right.\right.$ denotes the usual Sobolev space of all the functions $u \in L^{p}(\Omega)$ whose generalized derivatives for orders $|\mu| \leq m$ satisfy $D^{\mu} u=\partial_{1}^{\mu_{1}} \ldots \partial_{n}^{\mu_{n}} u \in L^{p}(\Omega)$. This space is equipped with its usual norm $\|\cdot\|_{W^{m, p}(\Omega)}$ and with the semi-norm

$$
|u|_{W^{m, p}(\Omega)}=\left\{\sum_{|\mu|=m} \int_{\Omega}\left|D^{\mu} u\right|^{p} \mathrm{~d} \boldsymbol{x}\right\}^{1 / p} .
$$

Throughout this paper the basic weight $\rho(r)$ is defined as

$$
\rho(r)=\left(1+|\boldsymbol{x}|^{2}\right)^{1 / 2}, \boldsymbol{x}=\left(x_{1}, \ldots, x_{n}\right) \in \mathbb{R}^{n},
$$

where $|\boldsymbol{x}|=\left(x_{1}^{2}+\ldots+x_{n}^{2}\right)^{1 / 2}$ is the distance to the origin. For any real numbers $\alpha$ and $p \geq 1, \mathcal{H}_{\alpha}^{m, p}(\Omega)$ is the space of all the functions $u \in L^{p}(\Omega)$ whose derivatives for orders $|\mu| \leq m$ satisfy

$$
\rho(r)^{\alpha+|\mu|} D^{\mu} u \in L^{p}(\Omega)
$$

The space $\mathcal{H}_{\alpha}^{m, p}(\Omega)$ is a Banach space when equipped with the norm

$$
\|u\|_{\mathcal{H}_{\alpha}^{m, p}(\Omega)}=\left(\sum_{|\mu| \leq m} \int_{\Omega} \rho(r)^{(\alpha+|\mu|) p}\left|D^{\mu} u\right|^{p} \mathrm{~d} \boldsymbol{x}\right)^{1 / p}
$$

Define also the space $\stackrel{\circ}{\mathcal{H}}_{\alpha}^{m, p}(\Omega)$ as the closure of $\mathscr{D}(\Omega)$ in $\mathcal{H}_{\alpha}^{m, p}(\Omega)$ and let $\mathcal{H}_{-\alpha}^{-m, p^{\prime}}(\Omega)$ be its dual. The reader can consult, e.g., $[10,22,25,29,30]$ for a detailed study of all these spaces. Notice that the local properties of the space $\mathcal{H}_{\alpha}^{m, p}$ coincide with those of the classical Sobolev space $W^{m, p}$.

In order to define the traces of functions of $\mathcal{H}_{\alpha}^{m, p}\left(\mathbb{R}_{+}^{n}\right)$, one extends the above definitions to real values of $m$. The reader can refer to [25] for such a definition which is dropped here for the sake of simplicity. We retain only that there exists a linear continuous trace mapping $\gamma=\left(\gamma_{0}, \gamma_{1}, \ldots, \gamma_{m-1}\right)$ from $\mathcal{H}_{\alpha}^{m, 2}\left(\mathbb{R}_{+}^{n}\right)(m \geq 1)$ to $\prod_{j=0}^{m-1} \mathcal{H}_{\alpha+j+1 / 2}^{m-j-\frac{1}{2}, 2}\left(\mathbb{R}^{n-1}\right)$ such that

$$
\forall u \in \mathscr{D}\left(\overline{\mathbb{R}}_{+}^{n}\right), \gamma u=\left(u\left(x^{\prime}, 0\right), \partial_{n} u\left(x^{\prime}, 0\right), \ldots, \partial_{n}^{m-1} u\left(x^{\prime}, 0\right)\right) .
$$

Moreover, for any $u \in \mathcal{H}_{\alpha-1}^{1,2}\left(\mathbb{R}_{+}^{n}\right), \forall v \in \mathcal{H}_{-\alpha}^{1,2}\left(\mathbb{R}_{+}^{n}\right)$ the following Green's formula holds

$$
\begin{aligned}
\int_{\mathbb{R}_{+}^{n}} \frac{\partial u}{\partial x_{i}} v \mathrm{~d} x & =-\int_{\mathbb{R}_{+}^{n}} u \frac{\partial v}{\partial x_{i}} \mathrm{~d} x, \quad i=1, \ldots, n-1, \\
\int_{\mathbb{R}_{+}^{n}} \frac{\partial u}{\partial x_{n}} v \mathrm{~d} x & =-\int_{\mathbb{R}_{+}^{n}} u \frac{\partial v}{\partial x_{n}} \mathrm{~d} x-\int_{\mathbb{R}^{n-1}} u\left(x^{\prime}, 0\right) v\left(x^{\prime}, 0\right) \mathrm{d} x^{\prime} .
\end{aligned}
$$


Now, let $\omega$ a bounded open set. We consider the space $V_{\alpha}^{m, p}(\omega)$ of all the functions $u$ defined a.e. in $\omega \backslash\{\mathbf{0}\}$ and whose derivatives for orders $|\mu| \leq m$ satisfy

$$
|\boldsymbol{x}|^{\alpha+|\mu|} D^{\mu} u \in L^{p}(\omega)
$$

This space is equipped with the norm

$$
\|u\|_{V_{\alpha}^{m, p}(\omega)}=\left(\sum_{|\mu| \leq m} \int_{\omega}|\boldsymbol{x}|^{(\alpha+|\mu|) p}\left|D^{\mu} u\right|^{p} \mathrm{~d} \boldsymbol{x}\right)^{1 / p} .
$$

We set

$$
|u|_{V_{\alpha}^{m, p}(\Omega)}=\left(\sum_{|\mu|=m} \int_{\omega}|\boldsymbol{x}|^{(\alpha+|\mu|) p}\left|D^{\mu} u\right|^{p} \mathrm{~d} \boldsymbol{x}\right)^{1 / p} .
$$

In this paper, the spaces $V_{\alpha}^{m, p}(\omega)$ are often considered when $\mathbf{0} \in \bar{\omega}$. In that case, the origin plays the role of a singular point. Notice that far from the origin the local properties of the space $V_{\alpha}^{m, p}(\omega)$ coincide topologically and algebraically with those of the Sobolev space $W^{m, p}(\omega)$.

The following imbedding is useful in this paper (see [13,31]): if $\frac{n}{p}-\frac{n}{q}+1>0$ and if $\beta+\frac{n}{q} \geq \alpha+\frac{n}{p}$, then

$$
\mathcal{H}_{\beta}^{1, q}\left(\mathbb{R}^{n}\right) \hookrightarrow \mathcal{H}_{\alpha}^{0, p}\left(\mathbb{R}^{n}\right) .
$$

When $p=2$, the spaces $W^{m, p}(\Omega), \mathcal{H}_{\alpha}^{m, p}(\Omega)$ and $V_{\alpha}^{m, p}(\omega)$ are written $H^{m}(\Omega), \mathcal{H}_{\alpha}^{m}(\Omega)$ and $V_{\alpha}^{m}(\omega)$, respectively.

A polynomial function $P$ belongs to $\mathcal{H}_{\alpha}^{m, p}\left(\mathbb{R}^{n}\right)$, if and only if

$$
\operatorname{deg} P<-\alpha-\frac{n}{p}
$$

Similarly, a polynomial $P$ belongs to $V_{\beta}^{m, p}(\omega)$, where $\omega$ is Lipschitz bounded domain and $\mathbf{0} \in \partial \omega$, if and only if

$$
\# P+\beta+\frac{n}{p}>0
$$

where \#P denotes the lowest degree of monomials of $P$. In particular, $\stackrel{\circ}{\mathbb{P}}_{k} \subset V_{\beta}^{m, p}(\omega)$ if

$$
\beta+\frac{n}{p}>-1
$$

In [2] it is proved that: if $u \in \mathcal{H}_{\alpha}^{1, p}\left(\mathbb{R}^{n}\right)$ and $\frac{n}{p}+\alpha \neq 0$ then

$$
\lim _{|x| \mapsto+\infty}|\boldsymbol{x}|^{\alpha+n / p}\|u(|\boldsymbol{x}|, .)\|_{L^{p}\left(S_{1}\right)}=0 .
$$

Here $S_{1}$ refer to the unit sphere of $\mathbb{R}^{n}$. In terms of the spaces $V_{\beta}^{1, p}$ this property can be written into the form

$$
\lim _{|\boldsymbol{x}| \mapsto 0}|\boldsymbol{x}|^{\beta+n / p}\|u(|\boldsymbol{x}|, .)\|_{L^{p}\left(S_{1}\right)}=0,
$$

if $u \in V_{\beta}^{1, p}(\omega), \omega$ being a bounded open set with $\mathbf{0} \in \partial \omega$, and $\beta+n / p \neq 0$. The latter property can be proved using (7) and Kelvin transform (see Sect. 3.3 and Prop. 1 hereafter). 
Remark 1. In the literature, many authors use the notation

$$
W_{\alpha}^{m, p}(\Omega)=\left\{u \in \mathscr{D}^{\prime}(\Omega) ; \rho^{|\mu|-m+\alpha} D^{\mu} u \in L^{p}(\Omega), \forall|\mu| \leq m\right\}
$$

instead of the notation $\mathcal{H}_{\alpha}^{m, p}(\Omega)$ which we prefer here for convenience. Of course, the identity $W_{\alpha}^{m, p}(\Omega)=$ $\mathcal{H}_{\alpha-m}^{m, p}(\Omega)$ allows the comparison.

Remark 2. Another family of spaces which is often used for treating problems in infinite regions are homogeneous spaces $D^{m, p}$. Recall that $D^{m, p}(\Omega)$ is defined as the closure of $\mathscr{D}(\bar{\Omega})$ with respect to the semi-norm

$$
\|u\|_{D^{m, p}(\Omega)}=\sum_{|\mu|=m}\left\|D^{\mu} u\right\|_{L^{p}(\Omega)} .
$$

The algebraic and topological identity (see [25])

$$
D^{m, p}\left(\mathbb{R}^{n}\right)=\mathcal{H}_{-m}^{m, p}\left(\mathbb{R}^{n}\right) \text { if } \frac{1}{p}-\frac{m}{n}>0
$$

shows that the spaces $D_{\alpha}^{m, p}$ are a particular case of the spaces $\mathcal{H}_{-m}^{m, p}\left(\mathbb{R}^{n}\right)$ (notice that the elements of $D^{m, p}$ are not necessarily distributions if $\frac{1}{p}-\frac{m}{n} \leq 0$. For example, $D^{m, 2}\left(\mathbb{R}^{n}\right) \not \subset \mathscr{D}^{\prime}\left(\mathbb{R}^{n}\right)$ if $n \leq 2 m$, see [27]).

As we said earlier, usual second and fourth order elliptic problems in unbounded regions of spaces can often lead to a problem of form (1), with $W$ a weighted space and $a(.,$.$) satisfying the classical assumptions of$ Lax-Milgram theorem. Let us sketch some illustrative examples:

Example 1 (the Laplace equation with a Dirichlet or a Neumann boundary condition). Let $\Omega$ be the half-space $\mathbb{R}_{+}^{n}=\left\{x_{n}>0\right\}$ or the exterior of a bounded Lipschitz open set $\omega$. Let us consider the Laplace problem:

Find $u \in \mathcal{H}_{-1}^{1}(\Omega)$ such that

$$
\Delta u=f \text { in } \Omega, u=0 \text { on } \partial \Omega,
$$

where $f \in \mathcal{H}_{1}^{-1}(\Omega)$ is given. The latter equation can be written into the variational form $(1)$ with $W^{\prime} \stackrel{\circ}{\mathcal{H}}_{-1}(\Omega)$ and

$$
a(u, v)=\int_{\Omega} \nabla u . \nabla v \mathrm{~d} \boldsymbol{x}, \ell(v)=\langle f, v\rangle .
$$

The linear form $\ell($.$) and the bilinear form a(.,$.$) are clearly continuous on \mathcal{H}_{-1}^{1}(\Omega)$. Using an adequate Hardy inequality (see [3] or [10]) we can prove that if $n \neq 2$ then

$$
\forall w \in \stackrel{\circ}{\mathcal{H}}_{-1}^{1}(\Omega), \int_{\Omega} \frac{w^{2}}{\rho^{2}} \mathrm{~d} \boldsymbol{x} \lesssim \int_{\Omega}|\nabla w|^{2} \mathrm{~d} \boldsymbol{x} .
$$

Hence if $n \neq 2, a(.,$.$) is coercive. Existence and Uniqueness of the solution stem from Lax-Milgram theorem.$ Moreover, it can be proven (see [22] when $\Omega$ is an exterior domain and [10] when $\Omega$ is the half-space) that if $f \in \mathcal{H}_{1}^{m}(\Omega)$ for some integer $m \geq 0$, then $u$ belongs to $\mathcal{H}_{-1}^{m+2}(\Omega)$ (the boundary of $\Omega$ is assumed sufficiently smooth).

The same results hold when $\Omega$ is whole the space $\mathbb{R}^{n}$ (see [22]).

Similarly, let us consider the Neumann problem:

Find $u \in \mathcal{H}_{-1}^{1}(\Omega)$ such that

$$
\Delta u=f \text { in } \Omega, \frac{\partial u}{\partial n}=g \text { on } \partial \Omega
$$


where $f \in \mathcal{H}_{1}^{0}(\Omega)$ and $g \in H^{-1 / 2}(\partial \omega)$ if $\Omega=\mathbb{R}^{n} \backslash \bar{\omega}$ and $g \in \mathcal{H}_{1 / 2}^{-1 / 2}\left(\mathbb{R}^{n-1}\right)$ if $\Omega=\mathbb{R}_{+}^{n}$. This problem is equivalent to a weak problem of the form (1) with $W=\mathcal{H}_{-1}^{1}(\Omega)$ and

$$
a(u, v)=\int_{\Omega} \nabla u . \nabla v \mathrm{~d} \boldsymbol{x}, \ell(v)=\int_{\Omega} f v+\langle g, v\rangle_{\partial \Omega} .
$$

Since the inequality (11) remains valid in the space $\mathcal{H}_{-1}^{1}(\Omega)$ (see the references cited above), this problem satisfies also the well-posedness assumptions of Lax-Milgram theorem (observe that the constants do not belong to the space $\left.\mathcal{H}_{-1}^{1}(\Omega)\right)$.

Remark 3. The above results on the Laplace equation can be extended to second order equations of the form

$$
\frac{\partial}{\partial x_{j}}\left(a_{i j}(\boldsymbol{x}) \frac{\partial u}{\partial x_{i}}\right)+c(\boldsymbol{x}) u=f \text { in } \Omega, u=0 \text { on } \partial \Omega,
$$

where the coefficients $a_{i j}$ and $c$ are such that

$$
\begin{aligned}
a_{i j} & \in L^{\infty}(\Omega), \\
a_{i j} \xi_{i} \xi_{j} & \geq \eta_{0}|\boldsymbol{\xi}|^{2}, \forall \boldsymbol{\xi}=\left(\xi_{1}, \ldots, \xi_{n}\right) \in \mathbb{R}^{n}, \\
-c_{0} \leq \rho^{2} c(\boldsymbol{x}) & \leq c_{1} \text { a.e. in } \Omega .
\end{aligned}
$$

Here $\eta_{0}>0, c_{0}>0$ and $c_{1} \geq 0$ are three constants with $c_{0}$ sufficiently small.

Remark 4. In studying the Laplace equation in unbounded domains, the case $n=2$ arises often as a critical case for which the weighted space $\mathcal{H}_{-1}^{1}(\Omega)$ is not really adequate (see $\left.[3,22]\right)$. Indeed, when $n=2$ it appears more convenient to use the modified space

$$
\mathcal{H}_{-1,0}^{1}(\Omega)=\left\{u \in \mathcal{D}^{\prime}(\Omega) ; \frac{u}{\rho(r) \log \left(2+r^{2}\right)} \in L^{2}(\Omega), \nabla u \in L^{2}(\Omega)\right\} .
$$

This particularity is inherent to Laplace equation itself (and also to some related problems) and to the functional framework. The method we propose here is general and is without any restriction on the dimension. However, to avoid fastidious notations, we consider only the spaces $\mathcal{H}_{\alpha}^{m, p}(\Omega)$. In fact, the addition of a logarithmic factor to the weight does not affect seriously the method and could be taken into account without major modifications. The author will treat this particularity in a forthcoming work.

Example 2 (a vector potential problem). Let $\boldsymbol{w} \in L^{2}\left(\mathbb{R}_{+}^{3}\right)^{3}$ such that

$$
\operatorname{div} \boldsymbol{w}=0 \text { in } \mathbb{R}_{+}^{3} .
$$

We look for a vector function $\boldsymbol{v} \in \mathcal{H}_{-1}^{1}\left(\mathbb{R}_{+}^{3}\right)^{3}$ satisfying

$$
\operatorname{curl} \boldsymbol{v}=\boldsymbol{w} \text { in } \mathbb{R}_{+}^{3}, \operatorname{div} \boldsymbol{v}=0 \text { in } \mathbb{R}_{+}^{3}, \boldsymbol{v} \cdot \boldsymbol{e}_{3}=0 \text { at } x_{3}=0 .
$$

Consider the space

$$
X\left(\mathbb{R}_{+}^{3}\right)=\left\{\boldsymbol{u} \in \mathcal{H}_{-1}^{1}\left(\mathbb{R}_{+}^{3}\right)^{3} ; \boldsymbol{u} . \boldsymbol{e}_{3}=0 \text { at } x_{3}=0\right\} .
$$

Problem (14) can be written into the form:

Find $\boldsymbol{v} \in X\left(\mathbb{R}_{+}^{3}\right)$ such that

$$
\int_{\mathbb{R}_{+}^{3}} \operatorname{curl} \boldsymbol{v} \cdot \operatorname{curl} \boldsymbol{u} \mathrm{d} \boldsymbol{x}+\int_{\mathbb{R}_{+}^{3}} \operatorname{div} \boldsymbol{v} \cdot \operatorname{div} \boldsymbol{u} \mathrm{d} \boldsymbol{x}=\int_{\mathbb{R}_{+}^{3}} \boldsymbol{w} \cdot \operatorname{curl} \boldsymbol{u} \mathrm{d} \boldsymbol{x},
$$


for each $\boldsymbol{u} \in X\left(\mathbb{R}_{+}^{3}\right)$. Indeed, it is quite clear that each solution of (14) is also a solution of (15). Conversely, let $\boldsymbol{v} \in X\left(\mathbb{R}_{+}^{3}\right)$ be a solution of (15). Then, choosing $\boldsymbol{u}=\nabla \varphi$ with $\varphi \in \mathcal{H}_{-2}^{2}\left(\mathbb{R}_{+}^{3}\right)$ solution of the Neumann problem (see [10])

gives $\operatorname{div} \boldsymbol{v}=0$. It follows that

$$
\Delta \varphi=\operatorname{div} \boldsymbol{v} \text { in } \mathbb{R}_{+}^{3}, \frac{\partial \varphi}{\partial x_{3}}=0 \text { at } x_{3}=0,
$$

$$
\int_{\mathbb{R}_{+}^{3}}(\operatorname{curl} \boldsymbol{v}-\boldsymbol{w}) \cdot \operatorname{curl} \boldsymbol{u} \mathrm{d} \boldsymbol{x}=0
$$

for each $\boldsymbol{u} \in X\left(\mathbb{R}_{+}^{3}\right)$. Hence,

$$
\operatorname{curl}(\operatorname{curl} \boldsymbol{v}-\boldsymbol{w})=\mathbf{0} \text { in } \mathscr{D}^{\prime}\left(\mathbb{R}_{+}^{3}\right)^{3}
$$

which implies that the vector function $\operatorname{curl} \boldsymbol{v}-\boldsymbol{w}$ belongs to the space

$$
H_{1}\left(\operatorname{curl} ; \mathbb{R}_{+}^{3}\right)=\left\{\varphi \in L^{2}\left(\mathbb{R}_{+}^{3}\right)^{3} ; \rho \operatorname{curl} \varphi \in L^{2}\left(\mathbb{R}_{+}^{3}\right)^{3}\right\} .
$$

Then, by means of the Green's formula (see [10])

$$
(\operatorname{curl} \boldsymbol{\varphi}, \boldsymbol{u})-(\boldsymbol{\varphi}, \operatorname{curl} \boldsymbol{u})=\left\langle\boldsymbol{\varphi} \wedge \boldsymbol{e}_{3}, \boldsymbol{u}\right\rangle_{x_{3}=0}
$$

which is valid for each $\boldsymbol{u} \in \mathcal{H}_{-1}^{1}\left(\mathbb{R}_{+}^{3}\right)^{3}$ and $\boldsymbol{\varphi} \in H_{1}\left(\operatorname{curl} ; \mathbb{R}_{+}^{3}\right)$, we get

$$
(\operatorname{curl} v-w) \times \boldsymbol{e}_{3}=\mathbf{0} \text { at } x_{3}=0 .
$$

Hence, the vector field $\boldsymbol{v}_{*}=\operatorname{curl} \boldsymbol{v}-\boldsymbol{w}$ satisfies

$$
\operatorname{curl} \boldsymbol{v}_{*}=\mathbf{0} \text { in } \mathbb{R}_{+}^{3}, \operatorname{div} \boldsymbol{v}_{*}=0 \text { in } \mathbb{R}_{+}^{3}, \boldsymbol{v}_{*} \times \boldsymbol{e}_{3}=\mathbf{0} \text { at } x_{3}=0 .
$$

Lemma 13 in [10] implies that $\boldsymbol{v}_{*}=\mathbf{0}$. We conclude that $\boldsymbol{v}$ is solution of (14).

Now, according to ([10], Cor. 8), the semi-norm

$$
\boldsymbol{u} \longrightarrow\left(\int_{\mathbb{R}_{+}^{3}}|\operatorname{curl} \boldsymbol{u}|^{2} \mathrm{~d} \boldsymbol{x}+\int_{\mathbb{R}_{+}^{3}}|\operatorname{div} \boldsymbol{u}|^{2} \mathrm{~d} \boldsymbol{x}\right)^{1 / 2}
$$

is a norm on the space $X\left(\mathbb{R}_{+}^{3}\right)$, equivalent to the norm $\|\cdot\|_{\mathcal{H}_{-1}^{1}\left(\mathbb{R}_{+}^{3}\right)^{3}}$. Consequently, the problem (15) admits one and only one solution in $X\left(\mathbb{R}_{+}^{3}\right)$, thanks to Lax-Milgram lemma.

Notice that the same results hold when $\Omega$ is an exterior domain (see $[20,21]$ ).

Example 3 (the biharmonic equation). In this last example, we consider the fourth order problem:

Find $u \in \mathcal{H}_{-2}^{2}\left(\mathbb{R}_{+}^{3}\right)$ such that

$$
\Delta^{2} u=f \text { in } \mathbb{R}_{+}^{3}, u=0 \text { at } x_{3}=0, \frac{\partial u}{\partial x_{3}}=0 \text { at } x_{3}=0,
$$

where $f \in \mathcal{H}_{2}^{-2}\left(\mathbb{R}_{+}^{3}\right)$. This problem can be written into the weak form:

Find $u \in \stackrel{\circ}{\mathcal{H}}_{-2}^{2}\left(\mathbb{R}_{+}^{3}\right)$ such that

$$
\int_{\mathbb{R}_{+}^{3}} \Delta u \cdot \Delta v \mathrm{~d} x=\langle f, v\rangle,
$$

for each $v \in \stackrel{\circ}{\mathcal{H}}_{0}^{2}\left(\mathbb{R}_{+}^{3}\right)$. It is proven in $[9,10]$ that the semi-norm

$$
v \longrightarrow\|\Delta v\|_{L^{2}\left(\mathbb{R}_{+}^{3}\right)},
$$


is a norm on the space $\stackrel{\circ}{\mathcal{H}}_{-2}^{2}\left(\mathbb{R}_{+}^{3}\right)$ which is equivalent to the norm $\|\cdot\|_{W_{-2}^{2}\left(\mathbb{R}_{+}^{3}\right)}$. We conclude that problem (17) admits one and only one solution $u$ and this solution depends continuously on the data.

Remark 5. The choice of the weight is crucial for getting a well posed variational formulation. In fact, the uniqueness of the solution can be lost if the weighted space contains polynomial functions (the case of the Neumann problem is detailed in Sect. 6). Similarly, the existence of strongly decreasing (at infinity) solutions could be lost, unless some compatibility conditions are satisfied by the data. However, there is often an intermediate case for which existence and uniqueness hold without any condition. In this case, the problem can in general be written in a weak form satisfying the assumptions of classical theorems (Lax-Milgram theorem, Babuska-Brezzi theorem, etc.). This is often the case for which the solution has also an integral representation. The reader can look at the case of the Laplace equation with a Neumann condition treated in Proposition 4 hereafter.

\section{INVERTED ELEMENT METHOD}

The purpose of this section is to expose the main ideas of the Inverted Finite Element Method. In the remaining of this paper, $\Omega$ denotes an unbounded domain. Some geometrical assumptions on $\Omega$ are made in Section 3.2 hereafter.

Consider a continuous problem written into the abstract form:

Find $u \in W$ such that

$$
\forall v \in W, a(u, v)=\ell(v),
$$

where $W$ is a given space. Here, we suppose that $W$ is a closed sub-space of $\mathcal{H}_{\alpha}^{1}(\Omega)^{s}$ for some integer $s$, equipped with the induced norm. This is often the situation when one deals with elliptic equations of second order. Moreover, the bilinear form $a(.,$.$) and the linear form \ell$ are supposed continuous on $W$ and satisfying the ellipticity condition

$$
\forall v \in W, a(v, v) \geq \mu_{0}\|v\|_{\mathcal{H}_{\alpha}^{1}(\Omega)^{s}}^{2}, \quad\left(\text { with } \mu_{0}>0\right) .
$$

The Galerkin method for approximating $u$ consists in replacing problem (18) by the finite-dimensional problem:

Find $u_{h} \in W_{h}$ such that

$$
\forall v_{h} \in W_{h}, a\left(u_{h}, v_{h}\right)=\ell\left(v_{h}\right)
$$

where $W_{h}$ is finite-dimensional. For simplicity, we shall restrict ourselves to continuous Inverted Elements which are similar to the classical conforming finite elements. Namely, we require that

$$
W_{h} \subset W .
$$

The first idea for constructing the space $W_{h}$ is to divide the domain $\Omega$ into two sub-domains; a finite subdomain $\Omega_{0}$ and an infinite sub-domain $\Omega_{\infty}$. Then, a usual finite element method is used in $\Omega_{0}$, while the unbounded region $\Omega_{\infty}$ is transformed into a bounded region by means of an adequate (but necessarily singular) mapping. Naturally, it is tempting to consider $\Omega_{\infty}$ as the exterior region of a sphere, and to map it into the interior of sphere by means of a classical inversion of the form $\boldsymbol{x} \mapsto \frac{\boldsymbol{x}}{|\boldsymbol{x}|^{2}}$. The drawback of this choice is the difficulty of ensuring the exact continuity of the method. The use of curved elements near to the spherical intersection $\bar{\Omega}_{\infty} \cap \bar{\Omega}_{0}$ can be of course a solution to this problem. Here, we prefer another approach based on the notions of polygonal inversion and polygonal radius introduced in the next subsections. The objective is to ensure the exact conformity at the intersection $\bar{\Omega}_{\infty} \cap \bar{\Omega}_{0}$ which will be chosen polygonal. 


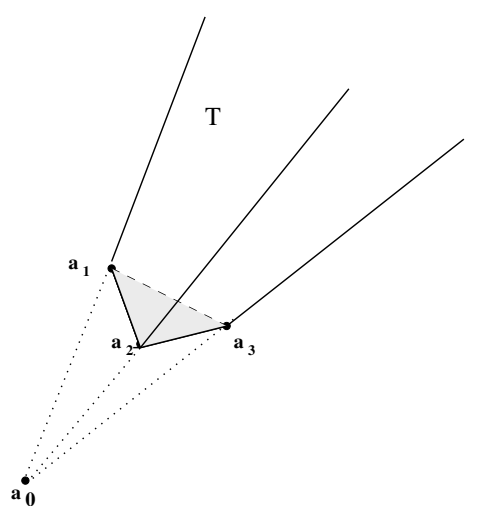

Figure 1. An example of a 3D infinite simplex.

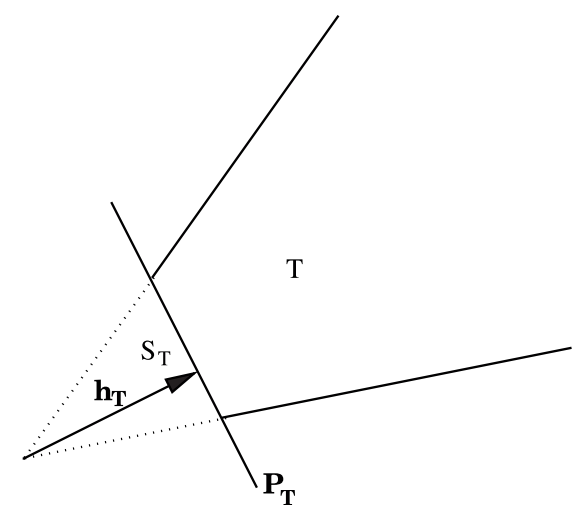

Figure 2. An illustration of the altitude vector, the finite simplex and the supporting hyperplane associated to a $2 \mathrm{D}$ infinite simplex.

\subsection{Some geometrical definitions and properties}

Definition 1. Let $\boldsymbol{a}_{j}=\left(a_{i j}\right)_{i=0}^{n}, j=0, \ldots, n$, be a collection of $n+1$ points of $\mathbb{R}^{n}$ which do not belong to the same hyperplane. Then, the infinite simplex whose vertices are $\boldsymbol{a}_{0}, \boldsymbol{a}_{1}, \ldots, \boldsymbol{a}_{n}$ is defined as

$$
T\left(\boldsymbol{a}_{0}, \boldsymbol{a}_{1}, \ldots, \boldsymbol{a}_{n}\right)=\left\{\boldsymbol{x}=\sum_{i=0}^{n} \lambda_{i} \boldsymbol{a}_{i}, \lambda_{0} \leq 0, \lambda_{i} \geq 0 \text { for } i=1, \ldots, n, \sum_{i=0}^{n} \lambda_{i}=1\right\} .
$$

Figures 1 and 2 show examples of infinite simplices when $n=3$ and when $n=2$, respectively.

The reference infinite and finite simplices are defined as

$$
\begin{gathered}
\hat{T}=\left\{\boldsymbol{x}=\left(\widehat{\lambda}_{1}, \ldots, \widehat{\lambda}_{n}\right) \in \mathbb{R}^{n}, \widehat{\lambda}_{k} \geq 0 \text { for } k=1, \ldots, n, \sum_{k=1}^{n} \widehat{\lambda}_{k} \geq 1\right\}, \\
\hat{K}=\hat{S}=\left\{\boldsymbol{x}=\left(\widehat{\lambda}_{1}, \ldots, \widehat{\lambda}_{n}\right) \in \mathbb{R}^{n}, 0 \leq \widehat{\lambda}_{k} \leq 1 \text { for } k=1, \ldots, n, \sum_{k=1}^{n} \widehat{\lambda}_{k} \leq 1\right\} .
\end{gathered}
$$

Now, let $T\left(\boldsymbol{a}_{0}, \boldsymbol{a}_{1}, \ldots, \boldsymbol{a}_{n}\right)$ be an infinite simplex. Then

- The vertices $\boldsymbol{a}_{1}, \ldots, \boldsymbol{a}_{n}$ are called the real vertices of $T$. 
- The vertex $\boldsymbol{a}_{0}$ is called the fictitious vertex of $T$.

- The finite simplex associated to $T$ is given by

$$
S_{T}\left(\boldsymbol{a}_{0}, \boldsymbol{a}_{1}, \ldots, \boldsymbol{a}_{n}\right)=\left\{\boldsymbol{x}=\sum_{i=0}^{n} \lambda_{i} \boldsymbol{a}_{i}, 0 \leq \lambda_{i} \leq 1,0 \leq i \leq n, \sum_{i=0}^{n} \lambda_{i}=1\right\} .
$$

- For each $i \in\{0, \ldots, n\}, \mathcal{P}^{i}(T)$ denotes the hyperplane containing the vertices $\left(\boldsymbol{a}_{j}\right)_{0 \leq j \leq n, j \neq i}$. The hyperplane $\mathcal{P}^{0}(T)$ will be called the supporting hyperplane of $T\left(\boldsymbol{a}_{0}, \boldsymbol{a}_{1}, \ldots, \boldsymbol{a}_{n}\right)$. Observe that $\boldsymbol{a}_{0} \notin \mathcal{P}^{0}(T)$.

- The $(n-1)$-faces of $T$ are defined as

$$
\Gamma_{i}(T)=T \cap \mathcal{P}^{i}(T), 0 \leq i \leq n .
$$

Note that $\Gamma_{1}(T), \ldots, \Gamma_{n}(T)$ are the unbounded faces of $T$ while $\Gamma_{0}(T)$ is the only bounded face of $T$. The face $\Gamma_{0}(T)$ is called the supporting face of $T$. More generally, for each integer $k$ such that $0 \leq k \leq n-2$, we call a $k$-face of $T$ a $k$-dimensional intersection of two $(k+1)$-faces of $T$. It follows that 0 -faces are nothing but the real vertices of $T$. A face of $T$ is a $k$-face of $T$ for some integer $0 \leq k \leq n-1$.

- The altitude vector of $T$, denoted by $\boldsymbol{h}_{T}$, is defined as $\boldsymbol{h}_{T}=\pi_{T} \boldsymbol{a}_{0}-\boldsymbol{a}_{0}$ where $\pi_{T} \boldsymbol{a}_{0}$ is the orthogonal projection of the fictitious vertex $\boldsymbol{a}_{0}$ on the supporting hyperplane $\mathcal{P}^{0}(T)$. Notice that $\left|\boldsymbol{h}_{T}\right|$ is the distance between $\boldsymbol{a}_{0}$ and $\mathcal{P}^{0}(T)$, and $\boldsymbol{h}_{T}$ is a vector normal to $\mathcal{P}^{0}(T)$ that points to the half-space containing $T$ (see Fig. 2).

- The quasi-inversion mapping associated to $T$ is defined as

$$
\begin{aligned}
\phi_{T}:\left(T \cup S_{T}\right) \backslash\left\{\boldsymbol{a}_{0}\right\} & \longrightarrow\left(T \cup S_{T}\right) \backslash\left\{\boldsymbol{a}_{0}\right\}, \\
\boldsymbol{x} & \mapsto \frac{\left|\boldsymbol{h}_{T}\right|^{4}}{\left[\left(\boldsymbol{x}-\boldsymbol{a}_{0}\right)^{t} \cdot \boldsymbol{h}_{T}\right]^{2}}\left(\boldsymbol{x}-\boldsymbol{a}_{0}\right)+\boldsymbol{a}_{0} .
\end{aligned}
$$

This mapping keeps the supporting face of $T$ invariant and transforms $T$ into $S_{T} \backslash\left\{\boldsymbol{a}_{0}\right\}$ and conversely. Moreover, $\phi_{T}$ is invertible and involutive (i.e. $\left.\phi_{T}^{-1}=\phi_{T}\right]$.

- We denote by $F_{T}$ the affine mapping which maps the reference infinite simplex $\hat{T}$ into $T$. The mapping $F_{T}$ maps also the reference simplex $\hat{S}$ into the finite simplex $S_{T}$ associated to $T$.

The altitude vector of reference infinite simplex $\hat{T}$ and its associated quasi-inversion are given by $\hat{\boldsymbol{h}}=n^{-1}(1, \ldots, 1)$ and $\hat{\phi}(\hat{\boldsymbol{x}})=\frac{1}{\left(\hat{\boldsymbol{x}}_{1}+\ldots+\hat{x}_{n}\right)^{2}} \hat{\boldsymbol{x}}$, respectively.

The following lemma is useful in the remaining of this paper.

Lemma 1. Let $T\left(\boldsymbol{a}_{0}, \ldots, \boldsymbol{a}_{n}\right)$ be an infinite simplex. Then,

$$
\begin{gathered}
F_{T}^{-1} \circ \phi_{T}=\hat{\phi} \circ F_{T}^{-1} . \\
\left|\operatorname{det}\left(\nabla \phi_{T}\right)\right|=\left(\frac{\left|\boldsymbol{h}_{T}\right|^{2}}{\left(\boldsymbol{x}-\boldsymbol{a}_{0}\right)^{t} \cdot \boldsymbol{h}_{T}}\right)^{2 n} .
\end{gathered}
$$

Proof.

1. We suppose without loss of generality that $\boldsymbol{a}_{0}=\mathbf{0}$. The affine mapping $F_{T}$ can be written in the form

$$
F_{T}(\hat{\boldsymbol{x}})=B \hat{\boldsymbol{x}} \text {, for any } \hat{\boldsymbol{x}} \in \hat{T} \cup \hat{S},
$$

where $B$ is a $n \times n$ constant matrix. The altitude vector $\boldsymbol{h}_{T}$ is given by

$$
\boldsymbol{h}_{T}=c_{T} B^{-t} \hat{\boldsymbol{h}} \text { with } c_{T}=\frac{|\hat{\boldsymbol{h}}|^{2}}{\left|B^{-t} \hat{\boldsymbol{h}}\right|^{2}} .
$$


Let $\boldsymbol{x} \in T \cup S_{T}-\{\mathbf{0}\}$ and set $\hat{\boldsymbol{x}}=F_{T}^{-1}(\boldsymbol{x})=B^{-1} \boldsymbol{x}$. Then,

$$
\phi_{T}(\boldsymbol{x})=\frac{\left|\boldsymbol{h}_{T}\right|^{4}}{\left[\boldsymbol{x}^{t} \cdot \boldsymbol{h}_{T}\right]^{2}} \boldsymbol{x}=\frac{\left|\boldsymbol{h}_{T}\right|^{4}}{c_{T}^{2}\left[\hat{\boldsymbol{x}}^{t} B^{t} \cdot B^{-t} \hat{\boldsymbol{h}}\right]^{2}} B \hat{\boldsymbol{x}}=\frac{|\hat{\boldsymbol{h}}|^{4}}{\left(\hat{\boldsymbol{x}}^{t} \cdot \hat{\boldsymbol{h}}\right)^{2}} B \hat{\boldsymbol{x}} .
$$

Hence,

which ends the proof of (21).

$$
F_{T}^{-1} \circ \phi_{T}(\boldsymbol{x})=B^{-1} \phi_{T}(\boldsymbol{x})=\frac{|\hat{\boldsymbol{h}}|^{4}}{\left(\hat{\boldsymbol{x}}^{t} . \hat{\boldsymbol{h}}\right)^{2}} \hat{\boldsymbol{x}}=\hat{\phi}(\hat{\boldsymbol{x}}),
$$

2. We have

$$
\begin{aligned}
\operatorname{det}\left(\nabla \phi_{T}\right) & =\operatorname{det}\left(\frac{\left|\boldsymbol{h}_{T}\right|^{4}}{\left(\boldsymbol{x}^{t} \cdot \boldsymbol{h}_{T}\right)^{2}} \boldsymbol{e}_{1}-2 \frac{\left|\boldsymbol{h}_{T}\right|^{4}}{\left(\boldsymbol{x}^{t} \cdot \boldsymbol{h}_{T}\right)^{3}} h_{1} \boldsymbol{x}, \ldots, \frac{\left|\boldsymbol{h}_{T}\right|^{4}}{\left(\boldsymbol{x}^{t} \cdot \boldsymbol{h}_{T}\right)^{2}} \boldsymbol{e}_{n}-2 \frac{\left|\boldsymbol{h}_{T}\right|^{4}}{\left(\boldsymbol{x}^{t} \cdot \boldsymbol{h}_{T}\right)^{3}} h_{n} \boldsymbol{x}\right) \\
& =\frac{\left|\boldsymbol{h}_{T}\right|^{4 n}}{\left(\boldsymbol{x}^{t} \cdot \boldsymbol{h}_{T}\right)^{2 n}} J_{n},
\end{aligned}
$$

where $h_{i}=\boldsymbol{h}_{T} \cdot \boldsymbol{e}_{i}, 1 \leq i \leq n$, and

$$
J_{n}=\operatorname{det}\left(\boldsymbol{e}_{1}-2 \frac{h_{1}}{\left(\boldsymbol{x}^{t} \cdot \boldsymbol{h}_{T}\right)} \boldsymbol{x}, \ldots, \boldsymbol{e}_{n}-2 \frac{h_{n}}{\left(\boldsymbol{x}^{t} \cdot \boldsymbol{h}_{T}\right)} \boldsymbol{x}\right) .
$$

Then, by induction we prove that

$$
J_{n}=-1,
$$

which ends the proof.

\subsection{The sub-division and the mesh of the domain}

We suppose that $\Omega$ satisfies the following geometrical assumption:

$(H)$ There exists at least two sub-domains $\Omega_{0}$ and $\Omega_{\infty}$ such that

$-\bar{\Omega}=\bar{\Omega}_{0} \cup \bar{\Omega}_{\infty}$;

- $\Omega_{0}$ is a bounded polygonal domain;

- $\Omega_{\infty}$ is unbounded and can be decomposed into the union of a finite number of infinite simplices $T_{1}, \ldots, T_{M}$ such that

1. $\bar{\Omega}_{\infty}=\cup_{\ell=1}^{M} T_{\ell}$;

2. $T_{1}, \ldots, T_{M}$ have the same fictitious vertex $\boldsymbol{a}_{0}$. Without loss of generality, we suppose that $\boldsymbol{a}_{0}=\mathbf{0}$;

3. for any $\ell, m \leq M$ with $\ell \neq m$, the intersection of $T_{\ell}$ and $T_{m}$ is either the empty set or a whole face.

The assumption $(H)$ is satisfied by most standard unbounded domains, namely the whole space, the half-space and the exterior regions of bounded (polygonal) domains (see Figs. 3 and 4 hereafter). Of course, such a decomposition is not unique in general.

For each $i \leq M$, we denote by $\boldsymbol{h}_{i}, \phi_{i}, S_{i}$ and $\Gamma_{i}$ respectively the altitude vector, the quasi-inversion, the finite simplex and the supporting face of $T_{i}$. The affine mapping which transforms the reference infinite simplex $\hat{T}$ into $T_{i}$ is denoted by $F_{i}$. We set

$$
\begin{aligned}
\Omega_{*} & =\frac{\circ}{\left(\cup_{i=1}^{M} S_{i}\right)}-\{\mathbf{0}\}, G_{i}=\left(T_{i} \cup S_{i}\right)-\{\mathbf{0}\}, i=1, \ldots, M, \\
\Gamma & =\bar{\Gamma}_{1} \cup \bar{\Gamma}_{2} \cup \ldots \cup \bar{\Gamma}_{M}=\bar{\Omega}_{*} \cap \bar{\Omega}_{\infty} .
\end{aligned}
$$

The polygonal domain $\Omega_{*}$ will be called the fictitious sub-domain of $\Omega$. It is important to distinguish it from $\Omega_{0}$.

We have

$$
\cup_{i=1}^{M} G_{i}=\bar{\Omega}_{*} \cup \bar{\Omega}_{\infty}-\{\mathbf{0}\} .
$$




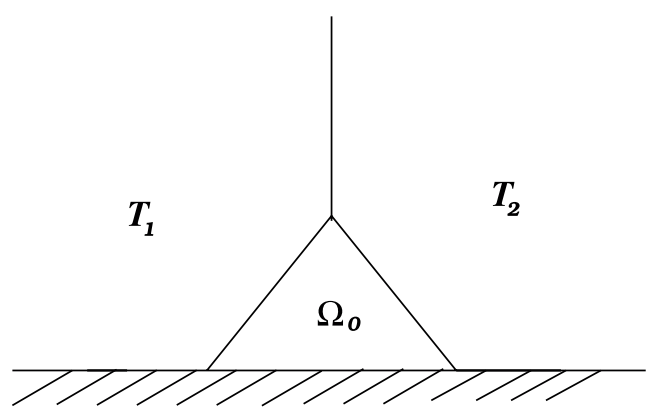

Figure 3 . The partition of the $2 \mathrm{D}$ half-space into the union of two infinite simplices $T_{1}$ and $T_{2}$ and a bounded region $\Omega_{0}$. Here $\bar{\Omega}_{0}=S_{1} \cup S_{2}-\{\mathbf{0}\}=\bar{\Omega}_{*}$, where $S_{1}$ and $S_{2}$ are the associated finite simplices.

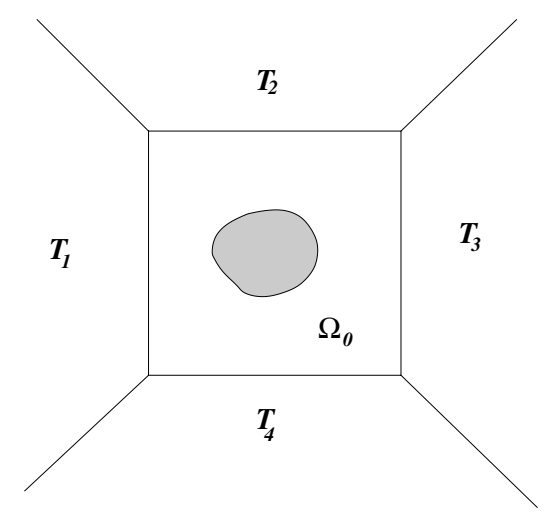

FiguRE 4 . The case of an exterior domain $\Omega=\mathbb{R}^{2}-\omega$. Here $\Omega$ is decomposed into the union of four finite simplices $T_{1}, T_{2}, T_{3}$ and $T_{4}$. We have $\bar{\Omega}_{*}=S_{1} \cup S_{2} \cup S_{3} \cup S_{4}$ and $\bar{\Omega}_{0}=\bar{\Omega}_{*}-\omega$.

\subsection{The polygonal inversion and polygonal Kelvin transform}

The objective of this section is to introduce some tools which will be used for defining the discrete space. Firstly, we consider the functions

$$
r_{i}(\boldsymbol{x})=\frac{\boldsymbol{x}^{t} \cdot \boldsymbol{h}_{i}}{\left|\boldsymbol{h}_{i}\right|^{2}}, i=1, \ldots, M .
$$

Then, the quasi-inversion mappings associated to the infinite simplices $T_{1}, \ldots, T_{M}$ are given by

$$
\phi_{i}(\boldsymbol{x})=r_{i}(\boldsymbol{x})^{-2} \boldsymbol{x} .
$$

Observe that

$$
r_{i}(\boldsymbol{x})=1 \text { if } \boldsymbol{x} \in \Gamma_{i} .
$$

Some simple but useful properties of these functions are stated in the following lemma.

Lemma 2. Let $i, j \in\{1, \ldots, M\}$ such that $G_{i} \cap G_{j} \neq \emptyset$. Then, for each $\boldsymbol{x} \in G_{i} \cap G_{j}$

$$
r_{i}(\boldsymbol{x})=r_{j}(\boldsymbol{x}), \quad \phi_{i}(\boldsymbol{x})=\phi_{j}(\boldsymbol{x}) .
$$

We have also

$$
r_{i}(\boldsymbol{x}) \simeq|\boldsymbol{x}| \text { in } G_{i}, i=1, \ldots, M \text {. }
$$


Proof. Suppose that $i \neq j$ and $G_{i} \cap G_{j} \neq \emptyset$. Let $\boldsymbol{a}_{1}, \ldots, \boldsymbol{a}_{k}(k \leq n)$ be the common real vertices of $T_{i}$ and $T_{j}$. We have on the one hand

$$
\boldsymbol{a}_{\ell} \cdot \boldsymbol{h}_{i}=\left|\boldsymbol{h}_{i}\right|^{2}, \boldsymbol{a}_{\ell} \cdot \boldsymbol{h}_{j}=\left|\boldsymbol{h}_{j}\right|^{2}, \forall 1 \leq \ell \leq k,
$$

since the points $\boldsymbol{a}_{1}, \ldots, \boldsymbol{a}_{k}$ belong to the supporting hyperplane of $T_{i}$.

On the other hand, any $\boldsymbol{x} \in G_{i} \cap G_{j}$ can be written into the form $\boldsymbol{x}=\sum_{\ell=1}^{k} \lambda_{\ell} \boldsymbol{a}_{\ell}$, with $\lambda_{\ell} \geq 0$ for each $\ell \leq k$ and $\sum_{\ell=1}^{k} \lambda_{\ell}>0$. Hence,

$$
\forall \boldsymbol{x} \in G_{i} \cap G_{j}, \quad \boldsymbol{x} . \boldsymbol{h}_{i}=\left(\sum_{\ell=1}^{k} \lambda_{\ell}\right)\left|\boldsymbol{h}_{i}\right|^{2}, \boldsymbol{x} . \boldsymbol{h}_{j}=\left(\sum_{\ell=1}^{k} \lambda_{\ell}\right)\left|\boldsymbol{h}_{j}\right|^{2} .
$$

Thus,

$$
r_{i}(\boldsymbol{x})=\sum_{\ell=1}^{k} \lambda_{\ell}=r_{j}(\boldsymbol{x})
$$

and $\phi_{i}(\boldsymbol{x})=\phi_{j}(\boldsymbol{x})$. This ends the proof of (24).

Now, let $\boldsymbol{a}_{1}, \ldots, \boldsymbol{a}_{n}$ be the real vertices of $T_{i}$ and $\boldsymbol{h}_{i}$ its altitude vector. Let $\boldsymbol{x}=\sum_{\ell=1}^{n} \lambda_{\ell} \boldsymbol{a}_{\ell} \in G_{i}$. Then, $r_{i}(\boldsymbol{x})=\sum_{\ell=1}^{n} \lambda_{\ell}$ and

$$
\left|\boldsymbol{h}_{i}\right| r_{i}(\boldsymbol{x}) \leq|\boldsymbol{x}| \leq \max _{1 \leq \ell \leq n}\left|\boldsymbol{a}_{\ell}\right| \sum_{\ell=1}^{n} \lambda_{\ell}=c r_{i}(\boldsymbol{x})
$$

which gives (25).

With help of assertions of Lemma 2 one can define the polygonal radius $r(\boldsymbol{x})$ in $\overline{\Omega_{\infty}} \cup \bar{\Omega}_{*}$ as follows

$$
r(\boldsymbol{x})=r_{i}(\boldsymbol{x}) \text { if } \boldsymbol{x} \in G_{i} .
$$

The polygonal inversion associated to the $T_{1}, \ldots, T_{M}$ is defined on $\cup_{i=1}^{M} G_{i}$ by

$$
\forall \boldsymbol{x} \in G, \phi(\boldsymbol{x})=\phi_{i}(\boldsymbol{x}), \quad \forall i \in\{1, \ldots, M\} .
$$

According to Lemma 2, we have

- the function $r(\boldsymbol{x})$ is globally continuous on $\bar{\Omega}_{\infty} \cup \bar{\Omega}_{*}-\{\mathbf{0}\}$ and is $\mathcal{C}^{\infty}$ in each sub-domain $G_{i}$;

$-r(\boldsymbol{x}) \simeq|\boldsymbol{x}|$ in $\bar{\Omega}_{\infty} \cup \bar{\Omega}_{*}-\{\mathbf{0}\}$

$-r(\boldsymbol{x})=1$ for each $\boldsymbol{x} \in \Gamma=\cup_{i=1}^{M} \Gamma_{i}$;

$-r(\boldsymbol{x}) \cdot r(\phi(\boldsymbol{x}))=1$;

- $\phi$ is continuous and one to one from $\Omega_{\infty}$ into $\Omega_{*}$ and $\phi^{-1}=\phi$. Moreover, $\phi$ preserves the points of the polygonal interface $\Gamma$ since $r(\boldsymbol{x})=1$ if $\boldsymbol{x} \in \Gamma$.

Given a real number $\gamma$, we define the polygonal Kelvin transform $\Lambda_{\gamma}$ as the operator which assigns to each function $u$ defined on $\bar{\Omega}_{\infty} \cup \bar{\Omega}_{*}-\{\mathbf{0}\}$ the function $\Lambda_{\gamma} u$ defined on the same set by

$$
\left(\Lambda_{\gamma} u\right)(\boldsymbol{x})=r(\boldsymbol{x})^{-\gamma} u(\phi(\boldsymbol{x})) .
$$

Observe that

$$
u(\boldsymbol{x})=r(\phi(\boldsymbol{x}))^{\gamma} \Lambda_{\gamma} u(\phi(\boldsymbol{x})) .
$$

Hence,

$$
u(\boldsymbol{x})=\left(\Lambda_{\gamma} u\right)(\boldsymbol{x}) \text { if } \boldsymbol{x} \in \Gamma .
$$

The next proposition describes the image of the weighted space $\mathcal{H}_{\alpha}^{m, p}\left(\Omega_{\infty}\right)$ by the operator $\Lambda_{\gamma}$. 
Proposition 1. Let $\alpha, \gamma$ and $p$ be three real numbers with $1<p<+\infty$. Let $u$ be a function defined in $\Omega_{\infty}$. We set

$$
\delta=\gamma-\alpha-2 \frac{n}{p} .
$$

1. If $m \in\{0,1\}$ and $u \in \mathcal{H}_{\alpha}^{m, p}\left(\Omega_{\infty}\right)$ then $\Lambda_{\gamma} u \in V_{\delta}^{m, p}\left(\Omega_{*}\right)$. Moreover

$$
\|u\|_{\mathcal{H}_{\alpha}^{m, p}\left(\Omega_{\infty}\right)} \simeq\left\|\Lambda_{\gamma} u\right\|_{V_{\delta}^{m, p}\left(\Omega_{*}\right)} .
$$

2. If $m \geq 0$ and $u \in \mathcal{H}_{\alpha}^{m, p}\left(\Omega_{\infty}\right)$. Then, $\Lambda_{\gamma} u$ satisfies

$$
\Lambda_{\gamma} u_{\mid S_{i}} \in V_{\delta}^{m, p}\left(S_{i}\right), \text { for } i=1, \ldots, M,
$$

and

$$
\|u\|_{\mathcal{H}_{\alpha}^{m, p}\left(T_{i}\right)} \simeq\left\|\Lambda_{\gamma} u\right\|_{V_{\delta}^{m, p}\left(S_{i}\right)}, \quad \text { for } i=1, \ldots, M .
$$

The proof of this proposition is given in appendix A.

\subsection{The mesh of the FEM domain and the fictitious sub-domain}

The last step in constructing the discrete space $W_{h}$ is the mesh of the domain. More precisely, this consists in triangulating the bounded sub-domain $\Omega_{0}$ and the fictitious subdomain $\Omega_{*}$ separately. Thus, we consider two families of triangulations

1. A classical finite element triangulation $\left\{K ; K \in \mathcal{T}_{h}\right\}$ of the region $\Omega_{0}$ into the union of simplices $K$ satisfying the usual regularity conditions:

(a) the intersection of two adjacent simplices is a whole $k$-dimensional face, with $0 \leq k \leq n-1$, or is empty;

(b) there exists a constant $\sigma$ such that

$$
\forall K \in \mathcal{T}_{h}, \frac{h_{K}}{\varrho_{K}} \leq \sigma \text { for every } h,
$$

where $h_{K}$ denotes the diameter of $K$ and $\varrho_{K}$ the radius of the inscribed sphere in $K$.

The (classical) discretization parameter associated to this triangulation is defined as

$$
h=\max _{K \in \mathcal{T}_{h}} \operatorname{diam}(K) .
$$

2. A graded triangulation $\left\{K ; K \in \widetilde{\mathcal{T}}_{h}\right\}$ of the fictitious domain $\Omega_{*}$ satisfying the regularity conditions above and, in addition, the following refinement assumptions:

(a) for any $K \in \widetilde{\mathcal{T}}_{h}^{*}=\left\{K \in \widetilde{\mathcal{T}}_{h} ; \mathbf{0} \notin K\right\}$

$$
\begin{aligned}
h_{K} & \lesssim h d_{K}^{1-\mu}, \\
h^{1 / \mu} & \lesssim d_{K} .
\end{aligned}
$$

Here $d_{K}$ denotes the distance between the origin $\mathbf{0}$ and $K$, and $\mu>0$ is the gradation parameter which will be chosen subsequently;

(b) for any $K \in \widetilde{\mathcal{T}}_{h}-\widetilde{\mathcal{T}}_{h}^{*}$

$$
h_{K} \lesssim h^{1 / \mu}
$$

(c) for any $K \in \widetilde{\mathcal{T}}_{h}$, there exists $i \in\{1, \ldots, M\}$ such that $K \subset S_{i}$. 
The last condition means that each triangulation of $\Omega_{*}$ is a conforming union of triangulations of the sub-domains $S_{i}, i=1, \ldots, M$. Namely, we can write

$$
\widetilde{\mathcal{T}}_{h}=\cup_{i=1}^{M} \widetilde{\mathcal{T}}_{h}^{i}
$$

where for each $i \leq M, \widetilde{\mathcal{T}}_{h}^{i}$ is a graded triangulation of $S_{i}$. Moreover, if $S_{i} \cap S_{j} \neq \emptyset$ then the meshes $\widetilde{\mathcal{T}}_{h}^{i}$ and $\widetilde{\mathcal{T}}_{h}^{j}$ must have the same trace on the intersection $S_{i} \cap S_{j}$.

All the above assumptions are intrinsic to $\mathcal{T}_{h}$ or to $\widetilde{\mathcal{T}}_{h}$. In order to ensure that the tringulations $\mathcal{T}_{h}$ and $\widetilde{\mathcal{T}}_{h}$ induces the same trace on the interface $\Gamma=\bar{\Omega}_{\infty} \cap \bar{\Omega}_{0}=\bar{\Omega}_{\infty} \cap \bar{\Omega}_{*}$, we assume the following:

If $K_{1} \in \mathcal{T}_{h}$ and $K_{2} \in \widetilde{\mathcal{T}}_{h}$, then $K_{1} \cap K_{2} \cap \Gamma$ is either empty or a whole face of both of $K_{1}$ and $K_{2}$.

\subsection{The discrete space and the interpolation operator}

Now, we are in position to construct the discrete space corresponding to the mesh of the domain. This space depends mainly on

- the choice of the sub-domains $\Omega_{0}$ and $\Omega_{\infty}$;

- the mesh parameters $h, \mu$;

- the exponent $\gamma$ of the polygonal Kelvin transform;

- the degree $k$ of the shape functions.

Namely, given an integer $k \in \mathbb{N}^{*}$ and a parameter $\gamma$, we associate to the above mesh, the following finite dimensional space

$$
\begin{aligned}
& \mathcal{H}_{h, k, \gamma}(\Omega)=\left\{u \in \mathcal{C}^{0}(\bar{\Omega}) ; u_{\mid K} \in \mathbb{P}_{k}(K), \forall K \in \mathcal{T}_{h}\right. \\
& \left.\Lambda_{\gamma} u_{\mid K} \in \mathbb{P}_{k}(K), \forall K \in \widetilde{\mathcal{T}}_{h}^{*}, \quad \Lambda_{\gamma} u_{\mid K} \in \stackrel{\circ}{\mathbb{P}}_{k}(K), \forall K \in \widetilde{\mathcal{T}}_{h}-\widetilde{\mathcal{T}}_{h}^{*}\right\} .
\end{aligned}
$$

A main feature of the IFE method is to consider $h$ as the only discretization parameter. The parameters, $\mu, \gamma$ and the subdomains $\Omega_{0}, T_{0}, \ldots, T_{M}$ are a priori fixed. Since their choice has a serious influence on the quality of approximation, we shall call them the adjustment parameters. The choice of the adjustment parameters is not arbitrary since they are subject to some constraints. Most of these constraints come from the analysis of the best approximation error. The following lemma gives a first condition on $\gamma$ :

Lemma 3. Suppose that $1<p<+\infty$ and that $\alpha+\frac{n}{p}<1+\gamma$. Then,

$$
\mathcal{H}_{h, k, \gamma}(\Omega) \hookrightarrow \mathcal{H}_{\alpha}^{1, p}(\Omega)
$$

Proof. We set $\delta=\gamma-\alpha-2 \frac{n}{p}$. Then, $\delta+\frac{n}{p}>-1$. Let $u_{h} \in \mathcal{H}_{h, k, \gamma}(\Omega)$. Then, $\left(\Lambda_{\gamma} u_{h}\right)_{\mid K} \in V_{\delta}^{1, p}(K)$ for each $K \in \mathcal{T}_{h} \cup \widetilde{\mathcal{T}}_{h}$, thanks to (6). The continuity of the functions $u_{h}$ and $r($.$) implies that \Lambda_{\gamma} u_{h} \in V_{\delta}^{1, p}\left(\Omega_{*}\right)$. We deduce that $u_{h \mid \Omega_{\infty}} \in \mathcal{H}_{\alpha}^{1, p}\left(\Omega_{\infty}\right)$, thanks to Proposition 1. We have also $u_{h \mid \Omega_{0}} \in W^{1, p}\left(\Omega_{0}\right)$. Since $u_{h}(\boldsymbol{x})=\Lambda_{\gamma} u_{h}(\boldsymbol{x})$ if $\boldsymbol{x} \in \Gamma$, we conclude that $u_{h} \in \mathcal{H}_{\alpha}^{1, p}(\Omega)$.

In the sequel, we shall suppose that the condition

$$
\gamma>\alpha+\frac{n}{p}-1
$$

is always fulfilled.

It remains to define the interpolation operator corresponding to the space $\mathcal{H}_{h, k, \gamma}(\Omega)$. The first step consists in defining a family of local interpolation operators as follows: let $K$ be an element of $\mathcal{T}_{h}$ or $\widetilde{\mathcal{T}}_{h}$ and $\boldsymbol{a}_{K}^{0}, \ldots, \boldsymbol{a}_{K}^{n}$ 
its vertices and consider the set of nodes

$$
\Sigma_{K}=\left\{\boldsymbol{x}=\sum_{i=0}^{n} \lambda_{i} \boldsymbol{a}_{K}^{i} ; \sum_{i=0}^{n} \lambda_{i}=1 \text { and } \lambda_{i} \in\left\{0, \frac{1}{k}, \frac{2}{k}, \ldots, \frac{k-1}{k}, 1\right\}\right\} .
$$

We denote by $\Pi_{K}$ the local $\mathbb{P}_{k^{-}}$interpolation operator of type $(k)$ defined as follows: for each function $v \in \mathcal{C}^{0}(K)$, $\Pi_{K} v$ is the only element of $\mathbb{P}_{k}$ such that

$$
\Pi_{K} v(\boldsymbol{a})=v(\boldsymbol{a})
$$

for any vertex $\boldsymbol{a} \in \Sigma_{K}$.

If $K \in \widetilde{\mathcal{T}}_{h}-\widetilde{\mathcal{T}}_{h}^{*}(\mathbf{0} \in K)$, we consider also the local $\stackrel{\circ}{\mathbb{P}}_{k}$-interpolation operator $\stackrel{\circ}{\Pi}_{K}$ of type $(k)$ defined as: for each $v \in \mathcal{C}^{0}(K-\{\mathbf{0}\}), \Pi_{K} u$ is the only element of $\stackrel{\circ}{\mathbb{P}}_{k}$ such that

$$
\stackrel{\circ}{\Pi}_{K} v(\boldsymbol{a})=v(\boldsymbol{a})
$$

for any node $\boldsymbol{a} \in \Sigma_{K}-\{\mathbf{0}\}$.

Under these considerations, one can associate to each function $v \in \mathcal{C}_{\text {loc }}^{0}(\bar{\Omega})$ its global interpolant $v_{h}$ defined by

$$
\begin{aligned}
v_{h \mid K} & =\Pi_{K} v_{\mid K} \text { for each } K \in \mathcal{T}_{h}, \\
\left(\Lambda_{\gamma} v_{h}\right)_{\mid K} & =\Pi_{K}\left[\left(\Lambda_{\gamma} v\right)_{\mid K}\right] \text { for each } K \in \widetilde{\mathcal{T}}_{h}^{*},
\end{aligned}
$$$$
\left(\Lambda_{\gamma} v_{h}\right)_{\mid K}=\stackrel{\circ}{\Pi}_{K}\left[\left(\Lambda_{\gamma} v\right)_{\mid K}\right] \text { for each } K \in \widetilde{\mathcal{T}}_{h}-\widetilde{\mathcal{T}}_{h}^{*} \text {. }
$$

The global interpolation operator $\Pi_{h}$ is the operator which assigns to each function $v \in \mathcal{C}_{\text {loc }}^{0}(\bar{\Omega})$, its interpolant $v_{h}$. This interpolant is continuous, thanks to the construction above.

Notice that the interpolation operator $\Pi_{h}$ depends on the mesh and on parameters $k$ and $\gamma$.

Notice also that $\Pi_{h} v$ belongs to the space $\mathcal{H}_{h, k, \gamma}(\Omega)$ for each function $v \in \mathcal{C}_{\text {loc }}^{0}(\bar{\Omega})$. The objective of the next section is to derive an estimate of the interpolation error $v-\Pi_{h} v$.

\section{The Best Approximation ERror}

We state the following:

Theorem 1. Let $p, q>1, \alpha, \beta$ four real numbers such that

$$
\begin{gathered}
\frac{n}{p}-\frac{n}{q}+1>0 \text { and } \beta+\frac{n}{q} \geq \alpha+\frac{n}{p}, \\
\beta+\frac{n}{q}-1<\gamma<\beta+\frac{n}{q}, \\
k+1>\frac{n}{q} .
\end{gathered}
$$

Let

$$
\mu_{m}=\frac{\beta-\alpha+n\left(\frac{1}{q}-\frac{1}{p}\right)}{k+1-m+n\left(\frac{1}{p}-\frac{1}{q}\right)}, \quad \tau_{m}=\frac{\mu_{m}}{\mu}, \text { for } 0 \leq m \leq k .
$$

Then, for each function $u \in \mathcal{H}_{\beta}^{k+1, q}(\Omega)$ the following estimate holds

$$
\left\|u-\Pi_{h} u\right\|_{\mathcal{H}_{\alpha}^{1, p}(\Omega)} \lesssim h^{[k+n(1 / p-1 / q)] \min \left(1, \tau_{1}\right)}\|u\|_{\mathcal{H}_{\beta}^{k+1, q}\left(\Omega_{\infty}\right)}+h^{k+n(1 / p-1 / q)}\|u\|_{W^{k+1, q}\left(\Omega_{0}\right)} .
$$


Further, for any nonegative integer $m \leq k$, we have

$$
\begin{gathered}
\sum_{K \in \widetilde{\mathcal{T}}_{h}}\left\|u-\Pi_{h}^{\alpha} u\right\|_{\mathcal{H}_{\alpha}^{m, p}(K)} \lesssim h^{[k+1-m+n(1 / p-1 / q)] \min \left(1, \tau_{m}\right)}\|u\|_{\mathcal{H}_{\beta}^{k+1, q}\left(\Omega_{\infty}\right)} \\
\sum_{K \in \mathcal{T}_{h}}\left\|u-\Pi_{h}^{\alpha} u\right\|_{\mathcal{H}_{\alpha}^{m, p}(K)} \lesssim h^{k+1-m+n(1 / p-1 / q)}\|u\|_{\mathcal{H}_{\beta}^{k+1, q}\left(\Omega_{0}\right)} .
\end{gathered}
$$

Notice that condition (37) implies the injection $\mathcal{H}_{\beta}^{k+1, q}(\Omega) \subset \mathcal{H}_{\alpha}^{1, p}(\Omega)$, thanks to (5). The second condition (38) combined with the first one implies in particular that $\gamma>\alpha+\frac{n}{p}-1$, which ensures the conformity injection (35). The third condition (39) is imposed to ensure the inclusion

$$
\mathcal{H}_{\beta}^{k+1, q}(\Omega) \subset \mathcal{C}_{l o c}^{0}(\bar{\Omega})
$$

where $\mathcal{C}_{l o c}^{0}(\bar{\Omega})$ is the space of continuous functions on $\bar{\Omega}$. Note that the inclusion (44) stems from the inclusion $W^{k+1, q}(\omega) \hookrightarrow \mathcal{C}^{0}(\bar{\omega})$ which is valid when $\omega$ is a bounded domain with a Lipschitz continuous boundary.

As for the finite element method, the proof of Theorem 1 is based on an analysis of the local interpolation operators.

\section{Proof of TheOREM 1}

Throughout the proof, assumptions (37)-(39) are supposed valid.

First step. The local interpolation error. The next proposition gives an estimate of the interpolation error in each inverted element.

Proposition 2. Let $i \in\{1, \ldots, M\}$. Let $m \leq k$ be an integer, $1<p, q<\infty$, and $\delta, \theta$ two real numbers such that

$$
-1<\theta+\frac{n}{q}<0
$$

and

Let

$$
V_{\theta}^{k+1, q}(\hat{K}) \hookrightarrow V_{\delta}^{m, p}(\hat{K}) .
$$

$$
\mu_{m}=\frac{\delta-\theta+(1 / p-1 / q) n}{k+1-m+(1 / p-1 / q) n}, \tau_{m}=\frac{\mu_{m}}{\mu} .
$$

Then, for each function $u \in V_{\theta}^{k+1, q}(K)$ the following estimates hold

$$
\begin{gathered}
\left|u-\Pi_{K} u\right|_{V_{\delta}^{m, p}(K)} \lesssim h^{[k+1-m+n(1 / p-1 / q)] \min \left(1, \tau_{m}\right)}|u|_{V_{\theta}^{k+1, q}(K)} \text { if } K \in \widetilde{\mathcal{T}}_{h}^{*}, \\
\left|u-\stackrel{\circ}{\Pi}_{K} u\right|_{V_{\delta}^{m, p}(K)} \lesssim h^{[k+1-m+n(1 / p-1 / q)] \tau_{m}}|u|_{V_{\theta}^{k+1, q}(K)} \text { if } K \in \widetilde{\mathcal{T}}_{h}-\widetilde{\mathcal{T}}_{h}^{*},
\end{gathered}
$$

where, for each $K$, the operators $\Pi_{K}$ and $\stackrel{\circ}{\Pi}_{K}$ are defined as in Section 3.5.

Proof of Proposition 2. Let $K \in \widetilde{\mathcal{T}}_{h}$ be a fixed finite simplex and let $F_{K}$ the affine mapping which transforms the reference element $\hat{K}$ into $K$, and chosen such that $F_{K}(\mathbf{0})=\mathbf{0}$ if $\mathbf{0} \in K$. This mapping is of the form

$$
\hat{\boldsymbol{x}} \in \hat{K} \mapsto B_{K} \hat{\boldsymbol{x}}+\boldsymbol{a}_{K} \in K
$$

with $\boldsymbol{a}_{K}=\mathbf{0}$ if $K \in \widetilde{\mathcal{T}}_{h}-\widetilde{\mathcal{T}}_{h}^{*}$. If $\varrho_{K}($ resp. $\varrho$ ) denotes the radius of the inscribed sphere in $K($ resp. in $\hat{K})$. Then (see, e.g., [14])

$$
\left\|B_{K}\right\| \leq \frac{\operatorname{diam}(K)}{\varrho} \lesssim h_{K},\left\|B_{K}^{-1}\right\| \leq \frac{\operatorname{diam}(\hat{K})}{\varrho_{K}} \lesssim h_{K}^{-1} .
$$


Now, let $u$ be a function in $V_{\theta}^{k+1, q}(K)$ and set

$$
\hat{u}=u \circ F_{K}
$$

Two cases are distinguished:

Case 1. If $K \in \widetilde{\mathcal{T}}_{h}^{*}$. Then, Assumptions (33) and (34) give

$$
|\boldsymbol{x}| \simeq d_{K} \text { for } \boldsymbol{x} \in K
$$

Hence, for any integer $m$, we have

$$
\begin{aligned}
\left|u-\Pi_{K} u\right|_{V_{\delta}^{m, p}(K)}^{p} & =\sum_{|\mu|=m} \int_{K}|\boldsymbol{x}|^{p(\delta+m)}\left|D^{\mu}\left(u-\Pi_{K} u\right)\right|^{p} \mathrm{~d} x \\
& \lesssim d_{K}^{p(\delta+m)} \sum_{|\mu|=m} \int_{K}\left|D^{\mu}\left(u-\Pi_{K} u\right)\right|^{p} \mathrm{~d} x \\
& \lesssim d_{K}^{p(\delta+m)}\left\|B_{K}^{-1}\right\|^{m p}\left|\operatorname{det} B \| \hat{u}-\Pi_{\hat{K}} \hat{u}\right|_{W^{m, p}(\hat{K})}^{p} \\
& \lesssim d_{K}^{p(\delta+m)} h_{K}^{-m p+n}\left|\hat{u}-\Pi_{\hat{K}} \hat{u}\right|_{W^{m, p}(\hat{K})}^{p} \\
& \lesssim d_{K}^{p(\delta+m)} h_{K}^{-m p+n} \inf _{\hat{p} \in \mathbb{P}_{k}}\|\hat{u}-\hat{p}\|_{W^{k+1, p}(\hat{K})}^{p},
\end{aligned}
$$

where we used the identity $\Pi_{K} \hat{p}=\hat{p}$ for each $\hat{p} \in \mathbb{P}_{k}$. Using the classical inequality (see [17])

$$
\inf _{\hat{p} \in \mathbb{P}_{k}}\|\hat{u}-\hat{p}\|_{W^{k+1, p(\hat{K})}} \lesssim|\hat{u}|_{W^{k+1, q}(\hat{K})}
$$

and the inequality

$$
|\hat{u}|_{W^{k+1, q}(\hat{K})} \leq\left\|B_{K}\right\|^{k+1}\left|\operatorname{det}\left(B_{K}\right)\right|^{-1 / q}|u|_{W^{k+1, q}(K)},
$$

we get

$$
\left|u-\Pi_{K} u\right|_{V_{\delta}^{m, p}(K)} \lesssim d_{K}^{\delta+m} h_{K}^{k+1-m+(1 / p-1 / q) n}|u|_{W^{k+1, q}(K)} .
$$

Moreover,

$$
\begin{aligned}
\left|u-\Pi_{K} u\right|_{V_{\delta}^{m, p}(K)} & \lesssim d_{K}^{\delta+m} h_{K}^{k+1-m+(1 / p-1 / q) n}|u|_{W^{k+1, q}(K)} \\
& \lesssim d_{K}^{\delta+m-\theta-k-1} h_{K}^{k+1-m+(1 / p-1 / q) n}|u|_{V_{\theta}^{k+1, q}(K)} \\
& \lesssim d_{K}^{\left(\mu_{m}-\mu\right)\left[k+1-m+\left(\frac{1}{p}-\frac{1}{q}\right) n\right]} h^{k+1-m+(1 / p-1 / q) n}|u|_{V_{\theta}^{k+1, q}(K)}
\end{aligned}
$$

Finally, assumption (33) gives

$$
\left|u-\Pi_{K} u\right|_{V_{\delta}^{m, p}(K)} \lesssim h^{[k+1-m+n(1 / p-1 / q)] \min \left(\tau_{m}, 1\right)}|u|_{V_{\theta}^{k+1, q}(K)}
$$


Case 2. If $K \in \widetilde{\mathcal{T}}_{h}-\widetilde{\mathcal{T}}_{h}^{*}$. Then for each integer $s \leq m$

$$
\begin{aligned}
\left|u-\stackrel{\circ}{\Pi}_{K} u\right|_{V_{\delta}^{s, p}(K)}^{p}= & \sum_{|\mu|=s} \int_{K}|\boldsymbol{x}|^{p(\delta+s)}\left|D^{\mu}\left(u-\stackrel{\circ}{\Pi}_{K} u\right)\right|^{p} \mathrm{~d} \boldsymbol{x} \\
\leq & \sum_{|\mu|=s}\left\|\left.B_{K}^{-1}\left|\|^{p s}\right| \operatorname{det} B_{K}\left|\int_{\hat{K}}\right| B_{K} \hat{\boldsymbol{x}}\right|^{p(\delta+s)}\left|D^{\mu}\left(\hat{u}-\stackrel{\circ}{\Pi}_{\hat{K}} \hat{u}\right)\right|^{p} \mathrm{~d} \hat{\boldsymbol{x}}\right. \\
\leq & \left\|B_{K}^{-1}\right\|^{p s}\left\|B_{K}\right\|\left\|^{\max (0, s+\delta) p}\right\| B_{K}^{-1} \|\left.\right|^{-\min (0, s+\delta) p}\left|\operatorname{det} B_{K}\right| \\
& \times \sum_{|\mu|=s} \int_{\hat{K}}|\hat{\boldsymbol{x}}|^{p(\delta+s)}\left|D^{\mu}\left(\hat{u}-\stackrel{\circ}{\Pi}_{\hat{K}} \hat{u}\right)\right|^{p} \mathrm{~d} \hat{\boldsymbol{x}} .
\end{aligned}
$$

Thus,

$$
\left|u-\stackrel{\circ}{\Pi}_{K} u\right|_{V_{\delta}^{s, p}(K)}^{p} \lesssim h_{K}^{p \delta+n}\left|\hat{u}-\stackrel{\circ}{\Pi}_{\hat{K}} \hat{u}\right|_{V_{\delta}^{s, p}(\hat{K})}^{p} .
$$

Let $\hat{p}$ be an arbitrary element of $\stackrel{\circ}{\mathbb{P}}_{k}(\hat{K})$. Then, since $\stackrel{\circ}{\Pi}_{\hat{K}} \hat{p}=\hat{p}$, we get

$$
\begin{aligned}
\left\|\hat{u}-\stackrel{\circ}{\Pi}_{\hat{K}} \hat{u}\right\|_{V_{\delta}^{m, p}(\hat{K})} & =\|(\hat{u}-\hat{p})-\hat{\Pi}(\hat{u}-\hat{p})\|_{V_{\delta}^{m, p}(\hat{K})} \\
& \lesssim\left\|I-\stackrel{\circ}{\Pi}_{\hat{K}}\right\|_{\mathcal{L}\left(V_{\theta}^{k+1, q}(\hat{K}), V_{\delta}^{m, p}(\hat{K})\right)}\|\hat{u}-\hat{p}\|_{V_{\theta}^{k+1, q}(\hat{K})} .
\end{aligned}
$$

The extension of the Deny-Lions inequality (51) to the space $V_{\theta}^{k+1, q}(\hat{K}) / \stackrel{\circ}{\mathbb{P}_{k}}$, required at this stage, is given in the following proposition

Proposition 3. Let $k \geq 0,1<q<\infty$ and $\theta$ such that

$$
-1<\theta+\frac{n}{q}<0
$$

Then,

$$
\inf _{\substack{\circ \\ p \in \mathbb{P}_{k}}}\|u-p\|_{V_{\theta}^{k+1, q}(\hat{K})} \lesssim|u|_{V_{\theta}^{k+1, q}(\hat{K})} .
$$

We prove Proposition 3 afterward. Proposition 3 is used here as follows:

$$
\begin{aligned}
\left\|\hat{u}-\stackrel{\circ}{\Pi}_{\hat{K}} \hat{u}\right\|_{V_{\delta}^{m, p}(\hat{K})} & \lesssim\left(\sum_{|\nu|=k+1} \int_{\hat{K}}|\hat{\boldsymbol{x}}|^{q(\theta+k+1)}\left|D^{\nu} \hat{u}\right|^{q} \mathrm{~d} \hat{\boldsymbol{x}}\right)^{1 / q} \\
& \lesssim\left\|B_{K}\right\|^{k+1}\left|\operatorname{det}\left(B_{K}\right)\right|^{-1 / q}\left(\sum_{|\nu|=k+1} \int_{K}\left|B_{K}^{-1} \boldsymbol{x}\right|^{q(\theta+k+1)}\left|D^{\nu} u\right|^{q} \mathrm{~d} \boldsymbol{x}\right)^{1 / q} \\
& \lesssim\left\|B_{K}\right\|^{k+1-\min (0, \theta+k+1)}\left\|B_{K}^{-1}\right\|^{\max (0, \theta+k+1)}\left|\operatorname{det}\left(B_{K}\right)\right|^{-1 / q}|u|_{V_{\theta}^{k+1, q}(K)} \\
& \lesssim h_{K}^{-\theta-n / q}|u|_{V_{\theta}^{k+1, q}(K)},
\end{aligned}
$$

where we used (49). Replacing in (52) gives

$$
\left\|u-\stackrel{\circ}{\Pi}_{\hat{K}} u\right\|_{V_{\delta}^{m, p}(K)} \leq h_{K}^{\delta-\theta+n(1 / p-1 / q)}|u|_{V_{\theta}^{k+1, q}(K)} .
$$


With assumption (34) we get

$$
\left\|u-\stackrel{\circ}{\Pi}_{K} u\right\|_{V_{\delta}^{m, p}(K)} \lesssim h^{[k+1-m+n(1 / p-1 / q)] \mu_{m} / \mu}|u|_{V_{\theta}^{k+1, q}(K)} .
$$

This ends the proof of Proposition 2. It remains to prove Proposition 3 used here.

Proof of Proposition 3. The starting point is the following lemma (see [26,30]).

Lemma 4. (Hardy's inequality) Let $1<q<+\infty$ and $\theta \in \mathbb{R}-\{-1\}$. Let $f$ be a continuous function on $[0,+\infty[$ such that

$$
\int_{0}^{+\infty} t^{\theta+q}|f(t)|^{q} \mathrm{~d} t<+\infty
$$

Set

$$
F(t)= \begin{cases}-\int_{t}^{+\infty} f(t) \mathrm{d} t & \text { if } \theta>-1, \\ \int_{0}^{t} f(t) \mathrm{d} t & \text { if } \theta<-1 .\end{cases}
$$

Then,

$$
\int_{0}^{+\infty} t^{\theta}|F(t)|^{q} \mathrm{~d} t \leq\left(\frac{q}{|\theta+1|}\right)^{q} \int_{0}^{+\infty} t^{\theta+q}|f(t)|^{q} \mathrm{~d} t
$$

Now, For any real $\delta>0$, we set

$$
\hat{K}_{\delta}=(\hat{K} \cup \hat{T}) \cap B_{\delta}-\{0\},
$$

where $B_{\delta}$ is the ball of radius $\delta$. There exists $\delta_{0}>0$ sufficiently small such that

$$
\hat{K}_{\delta} \subset \hat{K} \text { if } \delta<\delta_{0} .
$$

Consider the space

We state the following:

$$
\stackrel{\circ}{V}_{\theta}^{k+1, q}\left(\hat{K}_{\delta}\right)=\left\{v \in V_{\theta}^{k+1, q}\left(\hat{K}_{\delta}\right) ; v=0 \text { on } \partial B_{\delta} \cap \hat{K}\right\}
$$

Lemma 5. Let $k \geq 0,0<\delta<\delta_{0}$, and suppose that

$$
\theta+\frac{n}{q} \notin\{-k, \ldots, 0\}
$$

Then, the semi-norm

$$
u \longmapsto|u|_{V_{\theta}^{k+1, q}\left(\hat{K}_{\delta}\right)}=\sum_{|\mu|=k+1}\left\|D^{\mu} u\right\|_{L^{q}\left(\hat{K}_{\delta}\right)},
$$

is a norm on the space $\stackrel{\circ}{V}_{\theta}^{k+1, q}\left(\hat{K}_{\delta}\right)$ which is equivalent to the norm $\|\cdot\|_{V_{\theta}^{k+1, q}\left(\hat{K}_{\delta}\right)}$.

Proof. Let $\varphi$ be a function in $\mathcal{C}^{\infty}\left(\overline{\hat{K}}_{\delta}\right) \cap{\stackrel{\circ}{V_{\theta}}}^{k+1, q}\left(\hat{K}_{\delta}\right)$. Then, necessarily $\varphi(\mathbf{0})=0$, since $|\boldsymbol{x}|^{\theta} \varphi \in L^{q}\left(\hat{K}_{\delta}\right)$ and $\theta+\frac{n}{q}<0$. For any $\boldsymbol{x} \in \hat{K}_{\delta}$, we set $\boldsymbol{x}=r \boldsymbol{\sigma}$ with $r \in[0, \delta]$ and $\boldsymbol{\sigma} \in S_{1}$. Here $S_{1}$ is unit sphere of $\mathbb{R}^{n}$. For each multi-index $\mu$ such that $|\mu| \leq k$ we set

$$
\varphi_{\mu}=D^{\mu} \varphi
$$

We have

$$
\varphi_{\mu}(\boldsymbol{x})=\varphi_{\mu}(r \boldsymbol{\sigma})=-\int_{r}^{\delta} D_{t} \varphi_{\mu}(t \boldsymbol{\sigma}) \mathrm{d} t=\int_{0}^{r} D_{t} \varphi_{\mu}(t \boldsymbol{\sigma}) \mathrm{d} t+\varphi_{\mu}(0)
$$


If $\theta+|\mu|+\frac{n}{q} \neq 0$, then

$$
\begin{aligned}
\int_{\hat{K}_{\delta}}|\boldsymbol{x}|^{q(\theta+|\mu|)}\left|D^{\mu} \varphi\right|^{q} \mathrm{~d} \boldsymbol{x} & =\int_{\hat{K}_{\delta}}|\boldsymbol{x}|^{q(\theta+|\mu|)}\left|\varphi_{\mu}\right|^{q} \mathrm{~d} \boldsymbol{x} \\
& =\int_{\omega}\left(\int_{0}^{\delta} r^{q(\theta+|\mu|)+n-1}\left|\varphi_{\mu}(r \boldsymbol{\sigma})\right|^{q} \mathrm{~d} r\right) \mathrm{d} \boldsymbol{\sigma}
\end{aligned}
$$

where $\omega=S_{1} \cap \hat{K}_{\delta}$. Using Hardy's inequality (55) leads to

$$
\begin{aligned}
\int_{\hat{K}_{\delta}}|\boldsymbol{x}|^{q(\theta+|\mu|)}\left|D^{\mu} \varphi\right|^{q} \mathrm{~d} \boldsymbol{x} & \lesssim \int_{\omega}\left(\int_{0}^{\delta} r^{q(\theta+|\mu|+1)+n-1}\left|D_{r} \varphi_{\mu}(r \sigma)\right|^{q} \mathrm{~d} r\right) \mathrm{d} \boldsymbol{\sigma} \\
& \lesssim \sum_{|\nu|=|\mu|+1} \int_{\hat{K}_{\delta}}|\boldsymbol{x}|^{q(\theta+|\mu|+1)}\left|D^{\nu} \varphi(\boldsymbol{x})\right|^{q} \mathrm{~d} \boldsymbol{x} .
\end{aligned}
$$

The proof of Lemma 5 is ended by induction and by the density of the space of all the functions $\varphi \in$ $\mathcal{C}^{\infty}\left(\overline{\hat{K}}_{\delta}\right) \cap \stackrel{\circ}{V}_{\theta}^{k+1, q}\left(\hat{K}_{\delta}\right)$ in $\stackrel{\circ}{V}_{\theta}^{k+1, q}\left(\hat{K}_{\delta}\right)$ (see $\left.[25,30,33]\right)$.

Lemma 6. Let $m \geq 1$ be an integer and $\theta$ and $\delta$ two real numbers such that

$$
-1<\theta+\frac{n}{q}<0, \quad \delta<\delta_{0}
$$

and set

$$
\mathcal{O}=\hat{K}-\hat{K}_{\delta / 2}
$$

Then, the semi-norm

$$
[u]_{V_{\theta}^{k+1, q}(\hat{K})}=|u|_{V_{\theta}^{k+1, q}(\hat{K})}+\sum_{1 \leq|\mu| \leq k+1}\left|\int_{\mathcal{O}} D^{\mu} u \mathrm{~d} \boldsymbol{x}\right|,
$$

is a norm on $V_{\theta}^{k+1, q}(\hat{K})$ equivalent to the norm $\|\cdot\|_{V_{\theta}^{k+1, q}(\hat{K})}$.

Proof. We prove the lemma by contradiction; suppose that there exists a sequence $\left(u_{m}\right)$ such that

$$
\forall m \geq 1,\left\|u_{m}\right\|_{V_{\theta}^{k+1, q}(\hat{K})}=1 \text { and }\left[u_{n}\right]_{V_{\theta}^{k+1, q}(\hat{K})} \leq \frac{1}{m}
$$

Since $V_{\theta}^{k+1, q}(\hat{K})$ is a reflexive Banach space, there exists a subsequence still denoted $\left(u_{m}\right)$ which converges weakly in $V_{\theta}^{k+1, q}(\hat{K})$ to an element $u$. The lower semi-continuity of the norm $[\cdot]_{V_{\theta}^{k+1, q}(\hat{K})}$ implies that $[u]_{V_{\theta}^{k+1, q}(\hat{K})}=0$. Hence $u=0$ since the condition $\theta+\frac{n}{q}<0$ yields $\mathbb{R} \cap V_{\theta}^{k+1, q}(\hat{K})=\{0\}$.

In the remaining part of this proof, the constants involved in the symbols $\lesssim$ and $\simeq$ (defined before) do not depend on $m$ and the functions.

Now, let $\xi_{0}$ and $\xi_{1}$ two functions of $\operatorname{class} \mathcal{C}^{\infty}$ on $\mathbb{R}^{n}$ such that

$$
\begin{aligned}
& \xi_{0}(\boldsymbol{x})+\xi_{1}(\boldsymbol{x})=1, \\
& \operatorname{supp}\left(\xi_{0}\right) \subset B_{\delta} \\
& \operatorname{supp}\left(\xi_{1}\right) \subset \mathbb{R}^{n}-B_{\delta / 2} .
\end{aligned}
$$


Then, for each $m$,

$$
u_{m}=u_{m}^{(1)}+u_{m}^{(2)} \text { with } u_{m}^{(1)}(\boldsymbol{x})=\xi_{0}(\boldsymbol{x}) u_{m}(\boldsymbol{x}), u_{m}^{(2)}(\boldsymbol{x})=\xi_{1}(\boldsymbol{x}) u_{m}(\boldsymbol{x}) .
$$

Notice that $u_{m}^{(2)}(\boldsymbol{x})=0$ on $B_{\delta / 2} \cap \hat{K}$. Hence,

$$
\left\|u_{m}^{(2)}\right\|_{V_{\theta}^{k+1, q}(\hat{K})} \simeq\left\|u_{m}^{(2)}\right\|_{V_{\theta}^{k+1, q}(\mathcal{O})} .
$$

Since $\operatorname{dist}(\mathbf{0}, \mathcal{O})>0$, the space $V_{\theta}^{k+1, q}(\mathcal{O})$ is isomorphic to $W^{k+1, q}(\mathcal{O})$. It follows that the sequence $u_{m}$ converges weakly to zero in $W^{k+1, q}(\mathcal{O})$. The compactness of the imbedding $W^{k+1, q}(\mathcal{O}) \hookrightarrow W^{k, q}(\mathcal{O})$ implies that $u_{m}$ converges strongly to zero in $W^{k, q}(\mathcal{O})$. Since in addition $\left|u_{m}\right|_{V_{\theta}^{k+1, q}(\hat{K})}$ tends to zero, we deduce that

$$
u_{m} \text { converges strongly to } 0 \text { in } V_{\theta}^{k+1, q}(\mathcal{O}) \text {. }
$$

Hence, $u_{m}^{(2)}$ converges strongly to 0 in $V_{\theta}^{k+1, q}(\mathcal{O})=W^{k+1, q}(\mathcal{O})$. Now, we deal with the sequence $\left(u_{m}^{(1)}\right)$. Since $u_{m}^{(1)}=0$ on $\partial B_{\delta} \cap \hat{K}_{\delta}$ and by virtue of Lemma 5 , we get

$$
\left\|u_{m}^{(1)}\right\|_{V_{\theta}^{k+1, q}(\hat{K})} \lesssim\left|u_{m}^{(1)}\right|_{V_{\theta}^{k+1, q}(\hat{K})} .
$$

But,

$$
\begin{aligned}
\left|u_{m}^{(1)}\right|_{V_{\theta}^{k+1, q}(\hat{K})}^{q}= & \sum_{|\mu|=k+1} \int_{\hat{K}_{\delta / 2}}|\boldsymbol{x}|^{q(\theta+k+1)}\left|D^{\mu} u_{m}^{(1)}\right|^{q} \mathrm{~d} \boldsymbol{x} \\
& +\sum_{|\mu|=k+1} \int_{\mathcal{O}}|\boldsymbol{x}|^{q(\theta+k+1)}\left|D^{\mu} u_{m}^{(1)}\right|^{q} \mathrm{~d} \boldsymbol{x} \\
\lesssim & \sum_{|\mu|=k+1} \int_{\hat{K}_{\delta / 2}}|\boldsymbol{x}|^{q(\theta+k+1)}\left|D^{\mu} u_{m}\right|^{q} \mathrm{~d} \boldsymbol{x} \\
& +\sum_{|\mu|=k+1} \int_{\mathcal{O}}|\boldsymbol{x}|^{q(\theta+k+1)}\left|D^{\mu} u_{m}^{(1)}\right|^{q} \mathrm{~d} \boldsymbol{x} .
\end{aligned}
$$

Hence,

It follows that

$$
\left\|u_{m}^{(1)}\right\|_{V_{\theta}^{k+1, q}(\hat{K})} \lesssim\left|u_{m}\right|_{V_{\theta}^{k+1, q}\left(\hat{K}_{\delta / 2}\right)}+\left\|u_{m}\right\|_{V_{\theta}^{k+1, q}(\mathcal{O})} .
$$

$$
u_{m}^{(1)} \text { converges strongly to } 0 \text { in } V_{\theta}^{k+1, q}(\hat{K})
$$

since $u_{m}$ converges strongly to zero in $V_{\theta}^{k+1, q}(\mathcal{O})$. We deduce that $u_{m}$ converges strongly to zero in $V_{\theta}^{k+1, q}(\hat{K})$ and we reach the contradiction with the equality $\left\|u_{m}\right\|_{V_{\theta}^{k+1, q}(\hat{K})}=1$.

We end the proof of Proposition 3 as follows; let $u \in V_{\theta}^{k+1, q}(\hat{K})$. Since $\theta+\frac{n}{q}>-1$, we have $\stackrel{\circ}{\mathbb{P}}_{k} \subset V_{\theta}^{k+1, q}(\hat{K})$. Moreover, for each $p \in \stackrel{\circ}{\mathbb{P}}_{k}$ we have

$$
\|u-p\|_{V_{\theta}^{k+1, q}(\hat{K})} \lesssim[u-p]_{V_{\theta}^{k+1, q}(\hat{K})} .
$$

Choosing $p \in \stackrel{\circ}{\mathbb{P}}_{k}$ such that

ends the proof.

$$
\forall 1 \leq|\mu| \leq k+1 \int_{\mathcal{O}} D^{\mu} p \mathrm{~d} x=\int_{\mathcal{O}} D^{\mu} u \mathrm{~d} x
$$


Second step. Global interpolation error. We are now in position to prove Theorem 1 . Let $k, p, q, \alpha$ and $\beta$ satisfying the assumptions of that theorem and let $u \in \mathcal{H}_{\beta}^{k+1, p}(\Omega)$. We have

$$
\left\|u-\Pi_{h} u\right\|_{\mathcal{H}_{\alpha}^{1, p}(\Omega)} \lesssim\left\|u-\Pi_{h} u\right\|_{W^{1, p}\left(\Omega_{0}\right)}+\sum_{i=1}^{M}\left\|u-\Pi_{h} u\right\|_{\mathcal{H}_{\alpha}^{1, p}\left(T_{i}\right)} .
$$

From the classical theory of the Finite element Method (see [14]), we know that

$$
\left\|u-\Pi_{h}^{\alpha} u\right\|_{W^{1, p}\left(\Omega_{0}\right)} \lesssim h^{k+n(1 / p-1 / q)}\|u\|_{W^{k+1, p}\left(\Omega_{0}\right)}
$$

On the other hand, Proposition 1 implies that for each $i \leq M$

$$
\begin{aligned}
\sum_{i=1}^{M}\left\|u-\Pi_{h} u\right\|_{\mathcal{H}_{\alpha}^{1, p}\left(T_{i}\right)} & \lesssim \sum_{i=1}^{M}\left\|\Lambda_{\gamma}\left(u-\Pi_{h} u\right)\right\|_{V_{\delta}^{1, p}\left(S_{i}\right)}^{p} \\
& \lesssim \sum_{K \in \mathcal{T}_{h}-\mathcal{T}_{h}^{*}}\left\|\Lambda_{\gamma} u-\Pi_{K}\left(\Lambda_{\gamma} u\right)\right\|_{V_{\delta}^{1, p}(K)}^{p}+\sum_{K \in \mathcal{T}_{h}^{*}}\left\|\Lambda_{\gamma} u-\Pi_{K}\left(\Lambda_{\gamma} u\right)\right\|_{V_{\delta}^{1, p}(K)}^{p}
\end{aligned}
$$

with $\delta=\gamma-\alpha-\frac{2 n}{p}$. Taking $\theta=\gamma-\beta-\frac{2 n}{q}$ in Proposition 2 leads to

$$
\begin{aligned}
\left\|u-\Pi_{h} u\right\|_{\mathcal{H}_{\alpha}^{1, p}\left(\Omega_{\infty}\right)} & \lesssim h^{[k+n(1 / p-1 / q)] \min \left(\tau_{1}, 1\right)} \sum_{i=1}^{M}\left|\Lambda_{\gamma} u\right|_{V_{\theta}^{k+1, q}\left(S_{i}\right)}, \\
& \lesssim h^{[k+n(1 / p-1 / q)] \min \left(\tau_{1}, 1\right)} \sum_{i=1}^{M}\|u\|_{\mathcal{H}_{\beta}^{k+1, q}\left(T_{i}\right)},
\end{aligned}
$$

where $\tau_{1}=\frac{\mu_{1}}{\mu}$ and

$$
\mu_{m}=\frac{\delta-\theta+n\left(p^{-1}-q^{-1}\right)}{k+1-m+n\left(p^{-1}-q^{-1}\right)}=\frac{\beta-\alpha+n\left(q^{-1}-p^{-1}\right)}{k+1-m+n\left(p^{-1}-q^{-1}\right)},
$$

for each $m \leq k$. This ends the proof of estimate (41). The second estimate (42) is also a direct consequence of Propositions 1 and 2 .

\section{Application to a model problem}

Let us come back to the model variational problem (18). Recall that $W$ is supposed to be a closed subspace of $\mathcal{H}_{\alpha}^{1}(\Omega)^{s}$ for some real $\alpha$ and some integer $s$, and equipped with the norm $\|\cdot\|_{\mathcal{H}_{\alpha}^{1}(\Omega)}(s=1$ if the problem is scalar, and $s=n$ if the problem involves vector fields). Consider also the corresponding discrete problem (19) with

$$
W_{h}=W \cap \mathcal{H}_{h, k, \gamma}(\Omega)^{s}
$$

and

$$
\gamma>\alpha+\frac{n}{2}-1
$$

The error $u-u_{h}$ can be estimated in the norm $\|\cdot\|_{\mathcal{H}_{\alpha}^{1}(\Omega)}$ using Céa's lemma

$$
\left\|u-u_{h}\right\|_{\mathcal{H}_{\alpha}^{1}(\Omega)^{s}} \lesssim \inf _{v_{h} \in W_{h}}\left\|u-v_{h}\right\|_{\mathcal{H}_{\alpha}^{1}(\Omega)^{s}}
$$


If we suppose in addition that $u$ is continuous, and that its interpolant $\Pi_{h} u$ is also in $W$, then we get

$$
\left\|u-u_{h}\right\|_{\mathcal{H}_{\alpha}^{1}(\Omega)^{s}} \lesssim\left\|u-\Pi_{h} u\right\|_{\mathcal{H}_{\alpha}^{1}(\Omega)^{s}} .
$$

Combining the latter with Theorem 1 yields

Theorem 2. Let $u \in W$ be a solution of the problem (18) and $u_{h}$ solution of the corresponding discrete problem (19). Suppose that u belongs to $\mathcal{H}_{\beta}^{k+1, q}(\Omega)$ for some real $\beta$ such that the Assumptions (38), (37) and (39) are fulfilled with $p=2$. Suppose also that $W$ is such that

$$
\forall v \in W \cap \mathcal{C}_{l o c}^{0}(\bar{\Omega})^{s}, \Pi_{h} v \in W .
$$

Then, the following estimate holds

$$
\left\|u-u_{h}\right\|_{\mathcal{H}_{\alpha}^{1}(\Omega)} \lesssim h^{[k+n(1 / 2-1 / q)] \min \left(1, \tau_{1}\right)}\|u\|_{\mathcal{H}_{\beta}^{k+1, q}\left(\Omega_{\infty}\right)}+h^{k+n(1 / 2-1 / q)}\|u\|_{W^{k+1, q}\left(\Omega_{0}\right)},
$$

with $\tau_{1}$ is given by (40) with $p=2$ and $m=1$. In particular, if $0<\mu<\mu_{1}$ then

$$
\left\|u-u_{h}\right\|_{\mathcal{H}_{\alpha}^{1}(\Omega)} \lesssim h^{k+n(1 / 2-1 / q)}\|u\|_{\mathcal{H}_{\beta}^{k+1}(\Omega)} .
$$

Remark 6. Making an analogy with the finite element method, one can suspect the weighted $L^{2}$ error $\left\|u-u_{h}\right\|_{\mathcal{H}_{\alpha}^{0}(\Omega)}$ to converge faster than the error $\left\|u-u_{h}\right\|_{\mathcal{H}_{\alpha}^{1}(\Omega)}$ (under some usual regularity assumptions). Unfortunately, an examination of this question reveals that the classical duality argument of the Aubin-Nietsche lemma (see, e.g., [14]) does not give any additional information on the convergence of the error $\left\|u-u_{h}\right\|_{\mathcal{H}_{\alpha}^{0}(\Omega)}$. The computational tests shown in Section 6.3 seem to confirm the absence of a superconvergence of the weighted $L^{2}$ error.

\section{Implementation of THE METHOD AND COMPUTATIONAL RESUlts}

To assess the validity of the method, we now proceed to use it for solving numerically a 3D test problem. The problem we choose here is the Laplace equation in the upper half-space of $\mathbb{R}_{+}^{3}$ with a Neumann boundary condition at $x_{3}=0$ : Find $u \in \mathcal{H}_{-1}^{1}\left(\mathbb{R}_{+}^{3}\right)$ solution of

$$
-\Delta u=f \text { in } \mathbb{R}_{+}^{3}, \frac{\partial u}{\partial x_{3}}=0 \text { at } x_{3}=0,
$$

where $f$ is given in $\mathcal{H}_{1}^{0}\left(\mathbb{R}_{+}^{3}\right)$. As it is underlined in Section 2, this problem admits one and only one solution and can be written into the variational form (see [10]): Find $u \in \mathcal{H}_{-1}^{1}\left(\mathbb{R}_{+}^{3}\right)$ such that

$$
\forall v \in \mathcal{H}_{-1}^{1}\left(\mathbb{R}_{+}^{3}\right), \int_{\mathbb{R}_{+}^{3}} \nabla u . \nabla v \mathrm{~d} \boldsymbol{x}=\int_{\mathbb{R}_{+}^{3}} f v \mathrm{~d} x .
$$

Given an integer $k \geq 0$, we denote by $\mathcal{N}_{k}^{\Delta}$ the space of harmonic polynomials of degree less than or equal to $k$ and whose normal derivative vanishes at $x_{3}=0$. When $k<0$ we set $\mathcal{N}_{k}^{\Delta}=\{0\}$. It can be easily proven that $\mathcal{N}_{k}^{\Delta}$ is the space of harmonic polynomials which are even with respect to the variable $x_{3}$.

The half-space satisfies clearly the geometrical assumption of Section 3.2. Consider an integer $k \geq 0$, a parameter $\gamma \in \mathbb{R}$ and a gradation parameter $\mu>0$, and define the discrete space $\mathcal{H}_{h, \gamma, k}\left(\mathbb{R}_{+}^{3}\right)$ as in Section 3.5. The inclusion $\mathcal{H}_{h, \gamma, k}\left(\mathbb{R}_{+}^{3}\right) \subset \mathcal{H}_{-1}^{1}\left(\mathbb{R}_{+}^{3}\right)$ holds if $\gamma>-1 / 2$.

The corresponding interpolation operator $\Pi_{h}$ satisfies clearly

$$
\forall v \in \mathcal{H}_{-1}^{1}\left(\mathbb{R}_{+}^{3}\right) \cap \mathcal{C}_{\text {loc }}^{0}\left(\overline{\mathbb{R}}_{+}^{3}\right) ; \quad \Pi_{h} v \in \mathcal{H}_{-1}^{1}\left(\mathbb{R}_{+}^{3}\right),
$$


for any real $\alpha$. We set $W_{h}=\mathcal{H}_{h, \gamma, k}\left(\mathbb{R}_{+}^{3}\right)$. The discrete problem associated to (61) is: find $u_{h} \in W_{h}$ such that

$$
\forall v \in W_{h}, \int_{\mathbb{R}_{+}^{3}} \nabla u_{h} \cdot \nabla v_{h} \mathrm{~d} x=\int_{\mathbb{R}_{+}^{3}} f v_{h} \mathrm{~d} x .
$$

We need the following result:

Proposition 4 (10]). Let $k \geq 0$ be an integer. For each $f$ in $\mathcal{H}_{s+1}^{0}\left(\mathbb{R}_{+}^{3}\right)$, the problem (61) has a solution in $\mathcal{H}_{s-1}^{1}\left(\mathbb{R}_{+}^{3}\right)$ if and only if $f$ satisfies

$$
\forall q \in \mathcal{N}_{s-1}^{\Delta},\langle f, q\rangle=0
$$

When it exists, the solution is unique up to an element of $\mathcal{N}_{-s-1}^{\Delta}$. Moreover, if $f \in \mathcal{H}_{s+1}^{k-1}\left(\mathbb{R}_{+}^{3}\right)$ for some integer $k \geq 1$, then $u \in \mathcal{H}_{s-1}^{k+1}\left(\mathbb{R}_{+}^{3}\right)$. In all the cases, the solution depends continuously on the data (with respect to the quotient norm).

Remark 7. Note on the one hand that if $s \leq 0$ then $\mathcal{N}_{s-1}^{\Delta}=\{0\}$ and the orthogonality condition (64) is trivial. On the other hand, if $s \geq 0$, then $\mathcal{N}_{-s-1}^{\Delta}=\{0\}$ and the uniqueness holds. The case $s=0$ corresponds to the well posed weak formulation (62) for which existence and uniqueness hold.

Combining Corollary 2 and Proposition 4 yields

Theorem 3. Suppose that $k \geq 1$. Let $u$ be a solution of (61) and $u_{h}$ solution of (63). If $f \in \mathcal{H}_{s+1}^{k-1}\left(\mathbb{R}_{+}^{3}\right)$ for some integer $s \geq 0$, and if $f$ satisfies the orthogonality condition (64). Then $u$ belongs to $\mathcal{H}_{s-1}^{k+1}\left(\mathbb{R}_{+}^{3}\right)$. If in addition

then

$$
s-\frac{1}{2}<\gamma<s+\frac{1}{2}
$$

where $\tau=\frac{s}{k \mu}$.

$$
\left\|u-u_{h}\right\|_{\mathcal{H}_{-1}^{1}\left(\mathbb{R}_{+}^{3}\right)} \lesssim h^{k \min (1, \tau)}\|u\|_{\mathcal{H}_{s-1}^{k+1}\left(\mathbb{R}_{+}^{3}\right)},
$$

Now, let us sketch some details concerning the numerical computation of some integrals appearing in the discretization of $(62)$.

\subsection{Sub-division of the domain and generation of the mesh}

As explained in Section 3.2, we decompose the half space into the union of a bounded domain $\Omega_{0}$ and three infinite simplices $T_{1}, T_{2}$ and $T_{3}$. We choose $\Omega_{0}=R \hat{\Omega}_{0}$ where $\hat{\Omega}_{0}$ is the tetrahedron whose vertices are $(1,0,0)$, $(\sqrt{3} / 2,-1 / 2,0),(-\sqrt{3} / 2,-1 / 2,0)$ and $(0,0,1)\left(R \hat{\Omega}_{0}\right.$ is the set of all the points $\boldsymbol{y}$ of the form $R \boldsymbol{x}$ with $\left.\boldsymbol{x} \in \hat{\Omega}_{0}\right)$. The parameter $R$ is fixed. The infinite simplices $T_{1}, T_{2}$ and $T_{3}$ are given by $T_{1}=R \hat{T}_{1}, T_{2}=R \hat{T}_{2}, T_{3}=R \hat{T}_{3}$ where $\hat{T}_{1}, \hat{T}_{2}$ and $\hat{T}_{3}$ are three infinite simplices whose real vertices are given by

$$
\begin{aligned}
& \hat{T}_{1}:(1,0,0),(\sqrt{3} / 2,-1 / 2,0),(0,0,1), \\
& \hat{T}_{2}:(\sqrt{3} / 2,-1 / 2,0),(-\sqrt{3} / 2,-1 / 2,0),(0,0,1), \\
& \hat{T}_{3}:(1,0,0),(-\sqrt{3} / 2,-1 / 2,0),(0,0,1) .
\end{aligned}
$$

Figure 5 gives an illustration of this decomposition.

Here $R$ is a free parameter which describes the size of the bounded domain $\Omega_{0}$. Namely, the smaller is $R$ the smaller is the finite elements region $\Omega_{0}$. However, inverted elements method does not use $R$ as a discretization parameter. In others terms, the bounded domain $\Omega_{0}$, the sub-domains $T_{1}, \ldots, T_{M}$, the parameters $\gamma$ and $\mu$ are $a$ priori fixed. According to Theorem 2, the error of approximation and the convergence of the method are, indeed, controlled by $h$ which is the unique discretization parameter. However, it could be interesting to investigate 


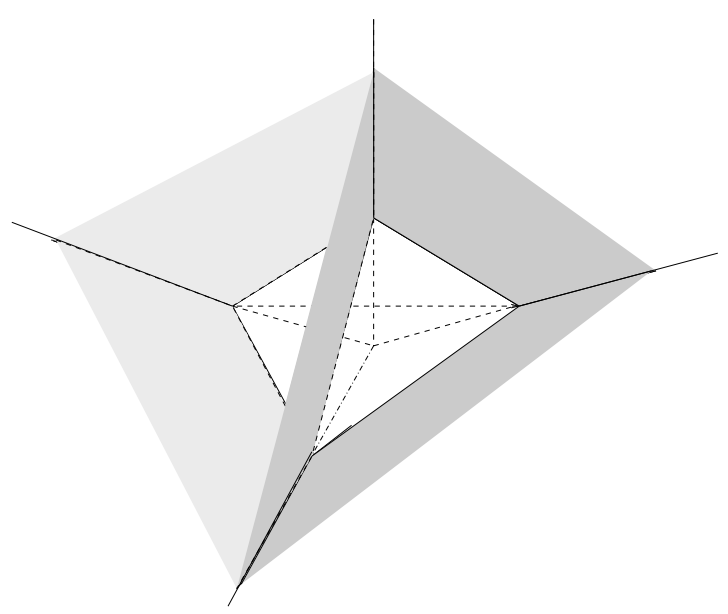

Figure 5. The decomposition of the upper half-space into the union of a finite simplex and three infinite simplices.

the influence of the adjustment parameters $\gamma, \mu$ and the radius of $\Omega_{0}$ on the convergence of the method. Recall that Theorem 2 suggests some restrictive indications on the choice of $\gamma$ and $\mu$. All these questions are discussed in Section 6.3 hereafter.

Now, let us display how the graded mesh of a tetrahedron could be generated.

For the sake of simplicity, we expose here the construction of this mesh in a two dimensional reference triangle $\hat{K}_{2}=\left\{(x, y) \in \mathbb{R}_{+} \times \mathbb{R}_{+} ; x+y \leq 1\right\}$. This construction remains valid in the $3 \mathrm{D}$ case in which we consider the test problem (62) and the forthcoming numerical results.

The first step consists to consider the increasing sequence $\left(\alpha_{i}\right)_{i \geq 1}$ defined by

$$
\alpha_{1}=1, \alpha_{i+1}=\alpha_{i}+\alpha_{i}^{1-\mu}
$$

Then, given an integer $N \geq 2$, we set $h_{N}=\alpha_{N}^{-\mu}$, and we consider the finite sequence

$$
d_{i}=\alpha_{i} h_{N}^{1 / \mu}, \quad 1 \leq i \leq N
$$

It is worth observing that $h_{N} \leq C_{\mu} N^{-1}$.

The next step consists to consider the segments

$$
D_{i}=\left\{(x, y) \in \hat{K}_{2} ; x+y=d_{i}\right\}, 1 \leq i \leq N
$$

Then, given an index $i \leq N$, we subdivide the segment $D_{i}$ into $i$ equal segments. The final mesh of $\hat{K}_{0}$ is obtained by linking the vertices as in Figure 6 . Observe that the diameter of a small triangle $K$, whose vertices are on the segments $D_{i}$ and $D_{i+1}$, satisfies $h_{K} \simeq d_{i+1}-d_{i}=\alpha_{i}^{1-\mu} h_{N}^{1 / \mu}=d_{i}^{1-\mu} h_{N}$.

Figure 6 shows the triangulations of $\hat{K}_{2}$ with $N=10$ and $\mu=1, \mu=0.5$ and $\mu=0.3$.

The method is extended without any serious difficulty to the three dimensional reference tetrahedron $\hat{S}$. The mesh of the three virtual simplices $S_{1}, S_{2}, S_{3}$ associated to the infinite simplices $T_{1}, T_{2}$ and $T_{3}$ is then obtained by mapping the mesh of $\hat{S}$ via an affine transformation. It is worth noting that the parameters $\mu$ and $N$ take the same values for $T_{1}, T_{2}$ and $T_{3}$. The mesh of the finite element domain $\Omega_{0}$ is obtained by a similar manner and with the same value of $N$ but without any gradation $(\mu=1)$. Note that $h \simeq R N^{-1}$. The total number of 

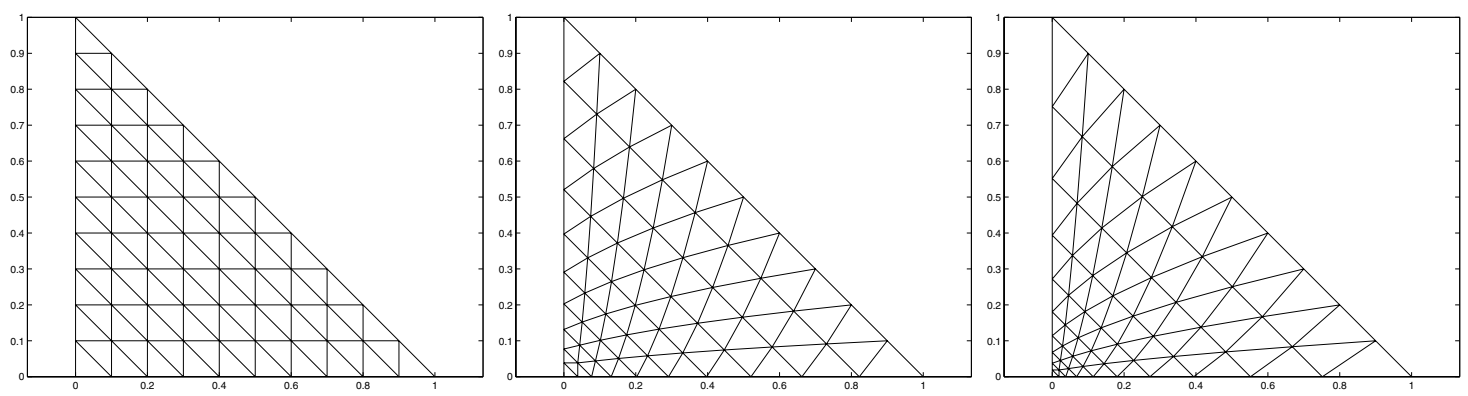

Figure 6 . The graded mesh of the reference simplex when $N=10 ; \mu=1$ (left) (in this case there is no gradation), $\mu=0.5$ (center), $\mu=0.3$ (right).

degrees of freedom (DOF) we get by such a construction in the case of the 3D half-space is

$$
D O F=\frac{2}{3}(N+1)(N+2)(N+3)-\left(3 N^{2}+5 N+3\right) \simeq \frac{2}{3} N^{3} .
$$

\subsection{Computation of the stiffness matrix}

Inspecting the various stages of the inverted element method, one can notice that its implementation is quite similar to that of the finite element method. In particular, it leads to a linear system with a sparse matrix. The only significant difference relies in the computation of the stiffness matrix which contain some additional but not fastidious complications. Our purpose here is to show how this matrix could be computed in practice.

Let $\left(M_{i}\right)_{1 \leq i \leq D O F}$ be the nodes of the total mesh. Define the family $\left(\psi_{i}\right)_{1 \leq i \leq D O F}$ of the basic functions of $W_{h}$ as

$$
\begin{aligned}
& -\psi_{i} \in W_{h} ; \\
& -\psi_{i}\left(M_{j}\right)=\delta_{i, j} \text { if } M_{j} \in K \text { for some } K \subset \Omega_{0} ; \\
& -\Lambda_{\gamma} \psi_{i}\left(M_{j}\right)=\delta_{i, j} \text { if } M_{j} \in K \text { for some } K \subset \Omega_{*} .
\end{aligned}
$$

Each element $u \in W_{h}$ can be decomposed into the form

$$
u(\boldsymbol{x})=\sum_{i=1}^{D O F} u_{i} \psi_{i}
$$

where the coefficients are given by

$$
u_{i}= \begin{cases}u\left(M_{i}\right) & \text { if } M_{j} \in K \text { for some } K \subset \Omega_{0}, \\ \left(\Lambda_{\gamma} u\right)\left(M_{i}\right) & \text { if } M_{i} \in K \text { for some } K \subset \Omega_{*} .\end{cases}
$$

The computation of the left hand side of (63) leads to the calculation of the matrix $A_{\gamma}$ whose coefficients are given by

$$
a_{i j}=\int_{\Omega}\left[\nabla \psi_{i}\right]^{t} \cdot \nabla \psi_{j} \mathrm{~d} \boldsymbol{x} .
$$

This matrix is sparse since $a_{i j}=0$ if the nodes $M_{i}$ and $M_{j}$ do not belong to the same element.

Further, we can write

$$
a_{i j}=\int_{\Omega_{0}}\left[\nabla \psi_{i}\right]^{t} \cdot \nabla \psi_{j} \mathrm{~d} \boldsymbol{x}+\sum_{m=1}^{M} \int_{T_{m}}\left[\nabla \psi_{i}\right]^{t} \cdot \nabla \psi_{j} \mathrm{~d} \boldsymbol{x} .
$$


The first term in this expression is a Finite Element term whose computation is quite easy. Hence, we deal only with the other terms which concern Inverted Elements. For each sub-domain $T_{m}, m=1, \ldots, M$, we have

$$
\begin{aligned}
\int_{T_{m}}\left[\nabla \psi_{i}\right]^{t} \cdot \nabla \psi_{j} \mathrm{~d} \boldsymbol{x} & =\sum_{K \subset S_{m}} \int_{\phi(K)}\left[\nabla \psi_{i}\right]^{t} \cdot \nabla \psi_{j} \mathrm{~d} \boldsymbol{x} \\
& =\sum_{K \subset S_{m}}\left|\operatorname{det}\left(B_{m}\right)\right| \int_{\left(F_{m}^{-1} \circ \phi\right)(K)}\left[\nabla_{\boldsymbol{\xi}} \tilde{\psi}_{i}\right]^{t} B_{m}^{-1} \cdot B_{m}^{-t} \cdot \nabla_{\boldsymbol{\xi}} \psi_{j} \mathrm{~d} \boldsymbol{\xi}
\end{aligned}
$$

where for each integer $i$,

$$
\tilde{\psi}_{i}(\boldsymbol{\xi})=\psi_{i}\left(F_{m}(\boldsymbol{\xi})\right), \boldsymbol{\xi} \in \hat{T} \text {. }
$$

Here $B_{m}$ the denotes the Jacobian matrix of the affine transformation $F_{m}$ which maps $\hat{T}$ into $T_{m}$. Now, we set $\hat{\psi}_{\ell}(\hat{\boldsymbol{x}})=\tilde{\psi}_{\ell}(\hat{\phi}(\hat{\boldsymbol{x}}))$ for $\ell=i$ or $\ell=j$. According to the chain rule, we have

$$
\left(\nabla_{\boldsymbol{\xi}} \tilde{\psi}_{\ell}\right) \circ \hat{\phi}(\hat{\boldsymbol{x}})=\left[J_{\boldsymbol{\xi}}\left(\hat{\phi}^{-1}\right) \circ \hat{\phi}(\hat{\boldsymbol{x}})\right] \nabla_{\hat{\boldsymbol{x}}} \hat{\psi}_{\ell}(\hat{\boldsymbol{x}})
$$

where $J_{\boldsymbol{\xi}}\left(\hat{\phi}^{-1}\right)=\left(\frac{\partial \hat{\phi}_{j}^{-1}}{\partial \xi_{i}}\right)_{1 \leq i, j \leq n}$ is the Jacobian matrix of $\hat{\phi}^{-1}$. Since $r(\hat{\boldsymbol{x}})=\hat{x}_{1}+\hat{x}_{2}+\hat{x}_{3}$ and $\hat{\phi}(\boldsymbol{x})=r(\hat{\boldsymbol{x}})^{-2} \hat{\boldsymbol{x}}$ we deduce that $\hat{\phi}^{-1}=\hat{\phi}$ and

$$
J_{\boldsymbol{\xi}}\left(\hat{\phi}^{-1}\right) \circ \hat{\phi}(\hat{\boldsymbol{x}})=r(\hat{\boldsymbol{x}})^{2}\left(I-2 c \hat{\boldsymbol{x}}_{*}^{t}\right)
$$

where $c=(1,1,1)^{t}$ and $\hat{\boldsymbol{x}}_{*}^{t}=\frac{1}{r(\hat{\boldsymbol{x}})} \hat{\boldsymbol{x}}$. Thus, $\operatorname{det}\left(J_{\boldsymbol{\xi}}\left(\hat{\phi}^{-1}\right)\right)=r(\hat{\boldsymbol{x}})^{-6}$ (see Lem. 1). It follows that for each tetrahedron $K \subset S_{m}$, we have

$$
\int_{\left(F_{m}^{-1} \circ \phi\right)(K)}\left[\nabla_{\boldsymbol{\xi}} \tilde{\psi}_{i}\right]^{t} B_{m}^{-1} \cdot B_{m}^{-t} \cdot \nabla_{\boldsymbol{\xi}} \tilde{\psi}_{j} \mathrm{~d} \boldsymbol{\xi}=\int_{F_{m}^{-1}(K)} r(\hat{\boldsymbol{x}})^{-2}\left[\nabla_{\hat{\boldsymbol{x}}} \hat{\psi}_{i}\right]^{t} A\left(\hat{\boldsymbol{x}}_{*}\right) \nabla_{\hat{\boldsymbol{x}}} \hat{\psi}_{j} \mathrm{~d} \hat{\boldsymbol{x}}
$$

where $A\left(\hat{\boldsymbol{x}}_{*}\right)$ is the symmetric matrix given by

$$
A\left(\hat{\boldsymbol{x}}_{*}\right)=\left(I-2 \hat{\boldsymbol{x}}_{*} \cdot c^{t}\right) B_{m}^{-1} \cdot B_{m}^{-t}\left(I-2 c \hat{\boldsymbol{x}}_{*}^{t}\right)
$$

Here, we used the identity $\hat{\phi} \circ F_{m}^{-1} \circ \phi=F_{m}^{-1}$ proved in Lemma 2.

The function $\hat{\psi}_{i}$ can be written into the form

$$
\hat{\psi}_{i}(\hat{\boldsymbol{x}})=r(\hat{\boldsymbol{x}})^{\gamma} w_{i}(\hat{\boldsymbol{x}})
$$

where $w_{i}$ is the only function of $\mathbb{P}_{k}\left(F_{m}^{-1}(K)\right)$ which satisfies $w_{i}\left(M_{i}\right)=1$ and $w_{i}(\hat{\boldsymbol{x}})=0$ if $\hat{\boldsymbol{x}}$ is another node of $F_{m}^{-1}(K)$. When $k=1$, this function is of the form $w_{i}(\boldsymbol{x})=a_{i}+\boldsymbol{\alpha}_{i}^{t} \cdot \hat{\boldsymbol{x}}$, where $a_{i} \in \mathbb{R}$ and $\boldsymbol{\alpha}_{i} \in \mathbb{R}^{3}$ are constants. It is worth noting that $a_{i}=0$ if $\mathbf{0} \in F_{m}^{-1}(K)$.

Consequently, the integral

$$
I_{K}=\int_{F_{m}^{-1}(K)} r(\hat{\boldsymbol{x}})^{-2}\left[\nabla \hat{\psi}_{i}\right]^{t} A\left(\hat{\boldsymbol{x}}_{*}\right) \nabla \hat{\psi}_{j} \mathrm{~d} \hat{\boldsymbol{x}}
$$

can be decomposed into three terms

$$
I_{K}=\sum_{s=1}^{3} I_{K, s}
$$


with

$$
\begin{aligned}
I_{K, 1}= & \int_{F_{m}^{-1}(K)} r(\hat{\boldsymbol{x}})^{2(\gamma-1)}\left\{\boldsymbol{\alpha}_{i}^{t} A\left(\hat{\boldsymbol{x}}_{*}\right) \boldsymbol{\alpha}_{j}+\gamma^{2}\left(\boldsymbol{\alpha}_{i}^{t} . \hat{\boldsymbol{x}}_{*}\right)\left(\boldsymbol{\alpha}_{j}^{t} . \hat{\boldsymbol{x}}_{*}\right) c^{t} A\left(\hat{\boldsymbol{x}}_{*}\right) c\right. \\
& \left.+\gamma\left(\boldsymbol{\alpha}_{j} . \hat{\boldsymbol{x}}_{*}\right) \boldsymbol{\alpha}_{i}^{t} A\left(\hat{\boldsymbol{x}}_{*}\right) c+\gamma\left(\boldsymbol{\alpha}_{i} . \hat{\boldsymbol{x}}_{*}\right) c^{t} A\left(\hat{\boldsymbol{x}}_{*}\right) \boldsymbol{\alpha}_{j}^{t}\right\} \mathrm{d} \hat{\boldsymbol{x}}, \\
I_{K, 2}= & \gamma \int_{F_{m}^{-1}(K)} r(\hat{\boldsymbol{x}})^{2 \gamma-3}\left\{a_{j} \boldsymbol{\alpha}_{i}^{t} A\left(\hat{\boldsymbol{x}}_{*}\right) c \mathrm{~d} \hat{\boldsymbol{x}}+a_{i} c^{t} A\left(\hat{\boldsymbol{x}}_{*}\right) \boldsymbol{\alpha}_{j} \mathrm{~d} \hat{\boldsymbol{x}}\right. \\
& \left.+\gamma a_{i}\left(\boldsymbol{\alpha}_{j}^{t} . \hat{\boldsymbol{x}}_{*}\right) c^{t} A\left(\hat{\boldsymbol{x}}_{*}\right) \cdot c \mathrm{~d} \hat{\boldsymbol{x}}+\gamma a_{j}\left(\boldsymbol{\alpha}_{i}^{t} . \hat{\boldsymbol{x}}_{*}\right) c^{t} A\left(\hat{\boldsymbol{x}}_{*}\right) c \mathrm{~d} \hat{\boldsymbol{x}}\right\} \\
I_{K, 3}= & \gamma^{2} a_{i} a_{j} \int_{F_{m}^{-1}(K)} r(\hat{\boldsymbol{x}})^{2 \gamma-4} c^{t} A\left(\hat{\boldsymbol{x}}_{*}\right) c \mathrm{~d} \hat{\boldsymbol{x}} .
\end{aligned}
$$

The expression of these integrals can be simplified since

$$
A\left(\hat{\boldsymbol{x}}_{*}\right) c=-\left(I-2 \hat{\boldsymbol{x}}_{*} c^{t}\right) B_{m}^{-1} B_{m}^{-t} c .
$$

Now, we consider the new variables

$$
\delta=r(\hat{\boldsymbol{x}})=\hat{x}_{1}+\hat{x}_{2}+\hat{x}_{3}, \sigma_{1}=\frac{\hat{x}_{1}}{\delta}, \sigma_{2}=\frac{\hat{x}_{2}}{\delta} .
$$

Notice that each one of the integrals $I_{K, s}, s=1, \ldots, 3$ is of the form

$$
\int_{F_{m}^{-1}(K)} r(\boldsymbol{x})^{\tau} f\left(\sigma_{1}(\hat{\boldsymbol{x}}), \sigma_{2}(\hat{\boldsymbol{x}})\right) \mathrm{d} \hat{\boldsymbol{x}}
$$

where $f$ is a polynomial function of total degree less than or equal to 2 . In order to compute these integrals we set

$$
\delta_{K}^{-}=\inf _{\boldsymbol{x} \in F_{m}^{-1}(K)} r(\boldsymbol{x}), \delta_{K}^{+}=\sup _{\boldsymbol{x} \in F_{m}^{-1}(K)} r(\boldsymbol{x}) .
$$

These two parameters are easy to compute numerically since their values are reached at one of the vertices of $F_{m}^{-1}(K)$. For each real $\delta$, such that $\delta_{K}^{-} \leq \delta \leq \delta_{K}^{+}$, we set

$$
Q_{K, \delta}=\left\{\left(\sigma_{1}, \sigma_{2}\right) \in \mathbb{R}^{2} ; \hat{\boldsymbol{x}}=\left(\delta \sigma_{1}, \delta \sigma_{2}, \delta\left(1-\sigma_{1}-\sigma_{2}\right)\right) \in F_{m}^{-1}(\bar{K})\right\} .
$$

The set $Q_{K, \delta}$ is convex and is in general either a vertex of the tetrahedron $F_{m}^{-1}(\bar{K})$ or an edge or a triangle or a quadrilateral (see Fig. 7).

The mapping $\left(\hat{x}_{1}, \hat{x}_{2}, \hat{x}_{3}\right) \mapsto\left(\delta, \sigma_{1}, \sigma_{2}\right)$ is clearly one to one from $\hat{S}$ onto $U=[0,|\hat{\boldsymbol{h}}|] \times \hat{K}_{2}=[0,1 / 3] \times \hat{K}_{2}$ where

$$
\hat{K}_{2}=\left\{\left(\sigma_{1}, \sigma_{2}\right) \in \mathbb{R}^{2} ; \sigma_{1} \geq 0, \sigma_{2} \geq 0, \sigma_{1}+\sigma_{2} \leq 1\right\}
$$

is the $2 \mathrm{D}$ reference triangle. It is also a diffeomorphism between their interiors. Hence, we can write

$$
\int_{F_{m}^{-1}(K)} r(\boldsymbol{x})^{\tau} f\left(\sigma_{1}(\hat{\boldsymbol{x}}), \sigma_{2}(\hat{\boldsymbol{x}})\right) \mathrm{d} \hat{\boldsymbol{x}}=\int_{\delta_{K}^{-}}^{\delta_{K}^{+}} \delta^{\tau+2}\left(\int_{Q_{K, \delta}} f\left(\sigma_{1}, \sigma_{2}\right) \mathrm{d} \sigma_{1} \mathrm{~d} \sigma_{2}\right) \mathrm{d} \delta
$$

The integrals

$$
\int_{Q_{K, \delta}} f\left(\sigma_{1}, \sigma_{2}\right) \mathrm{d} \sigma_{1} \mathrm{~d} \sigma_{2}
$$

are calculated exactly since $f\left(\sigma_{1}, \sigma_{2}\right)$ is a polynomial function of degree less than or equal 2. The integration with respect to the variable $\delta$ is done by means of a Gauss-Lobatto quadrature formula. 


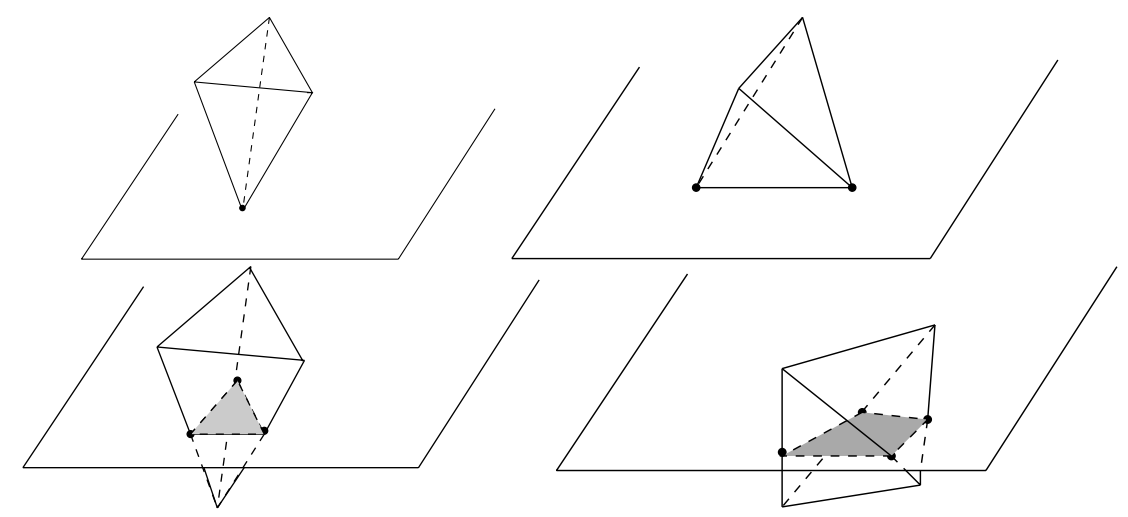

FIGURE 7. The intersection of a tetrahedron and a plane is either a vertex, an edge a triangle or a quadrilateral.

\subsection{Computational results}

In this section, we show some numerical experiments obtained with a 3D code written for solving the Neumann problem (61). The aim of these tests is to compare the numerical results with the predictions of Theorem 2 and to demonstrate the efficiency of the method. In particular, we study the dependence of the numerical error with respect to $h$. The influence of the adjustment parameters $\mu, \gamma$ and the radius of $\Omega_{0}($ here $R$ ) on the rate of convergence is also investigated. The implemented method corresponds to the case $k=1$ ( $\mathbb{P}^{1}$-IFEM).

Define the relative errors on $u$ and on its gradient with respect to the norms $\|\cdot\|_{\mathcal{H}_{-1}^{0}\left(\mathbb{R}_{+}^{3}\right)}$ and $\|\cdot\|_{L^{2}\left(\mathbb{R}_{+}^{3}\right)}$ as follows

$$
e_{0}=\frac{\left\|u-u_{h}\right\|_{\mathcal{H}_{-1}^{0}\left(\mathbb{R}_{+}^{3}\right)}}{\|u\|_{\mathcal{H}_{-1}^{0}\left(\mathbb{R}_{+}^{3}\right)}}, \quad e_{1}=\frac{\left|\nabla u-\nabla u_{h}\right|_{L^{2}\left(\mathbb{R}_{+}^{3}\right)}}{|\nabla u|_{L^{2}\left(\mathbb{R}_{+}^{3}\right)}} .
$$

We consider two examples for the tests

Example 1. $f=-\Delta u_{\epsilon}$ and $u=u_{\epsilon}$ where

$$
u_{\epsilon}(x, y, z)=\frac{z^{2}}{\left(1+x^{2}+y^{2}+z^{2}\right)^{\epsilon+2}} .
$$

Here $\epsilon$ is a fixed parameter on which depends the behavior at infinity. Notice that $f \in \mathcal{H}_{1}^{0}\left(\mathbb{R}_{+}^{3}\right)$ and $u \in \mathcal{H}_{-1}^{1}\left(\mathbb{R}_{+}^{3}\right)$ if $\epsilon>-3 / 4$. More generally, $f$ belongs to $\mathcal{H}_{\beta+2}^{0}\left(\mathbb{R}_{+}^{3}\right)$, while $u$ belongs to $\mathcal{H}_{\beta}^{2}\left(\mathbb{R}_{+}^{n}\right)$ for each $\beta<\frac{1}{2}+2 \epsilon$.

Example 2. We consider the problem (61) with $f=-\Delta u$, where

$$
u(x, y, z)=\frac{2}{\pi} \arctan \left(10 r^{2}\right)\left(\mathrm{e}^{-0.1 r^{2}}+\mathrm{e}^{-0.1(r-3)^{2}}\right) .
$$

Notice that $f \in \mathcal{H}_{\beta}^{0}\left(\mathbb{R}_{+}^{n}\right)$ and $u \in \mathcal{H}_{\beta}^{2}\left(\mathbb{R}_{+}^{n}\right)$ for any real $\beta$.

\subsubsection{Convergence of the method and influence of the parameter $\mu$}

In this paragraph we fix $\gamma=1$ and $R=1$ and we study the convergence of the error when $h \rightarrow 0$ and its dependence with respect to the parameter $\mu$. It is worth noting that the value of $R$ is taken relatively small in order to show that the method converges even if the FEM region $\Omega_{0}$ is small (the accuracy of the numerical errors $e_{0}$ and $e_{1}$ can be improved a little bit if $R$ is well chosen. See Paragraph 6.3.3).

In Table 1, we display the relative errors for several values of $h$ and $\mu$ when $\gamma=1$ and $R=1$ (Ex. 1). 
TABLE 1 . The relative errors $e_{0}$ and $e_{1}$ when $R=1$ and $\gamma=1$.

\begin{tabular}{|c|c|c|c|c|c|c|c|c|c|c|}
\hline \multirow[b]{3}{*}{$N$} & \multirow[b]{3}{*}{$h$} & \multirow[b]{3}{*}{ DOF } & \multicolumn{4}{|c|}{$\mu$} & \multicolumn{4}{|c|}{$\mu$} \\
\hline & & & 1 & 0.7 & 0.5 & 0.4 & 1 & 0.7 & 0.5 & 0.4 \\
\hline & & & \multicolumn{4}{|c|}{ The relative error $e_{0}$} & \multicolumn{4}{|c|}{ The relative error $e_{1}$} \\
\hline 10 & 0.30 & 791 & 0.328 & 0.255 & 0.220 & 0.204 & 0.866 & 0.720 & 0.565 & 0.481 \\
\hline 20 & 0.15 & 5781 & 0.207 & 0.130 & 0.087 & 0.076 & 0.654 & 0.359 & 0.205 & 0.158 \\
\hline 30 & 0.10 & 18971 & 0.146 & 0.070 & 0.040 & 0.038 & 0.463 & 0.204 & 0.104 & 0.082 \\
\hline 40 & 0.075 & 44361 & 0.107 & 0.041 & 0.025 & 0.026 & 0.345 & 0.133 & 0.067 & 0.054 \\
\hline 50 & 0.06 & 85951 & 0.081 & 0.028 & 0.020 & 0.020 & 0.270 & 0.095 & 0.049 & 0.040 \\
\hline 60 & 0.05 & 147741 & 0.064 & 0.022 & 0.018 & & 0.219 & 0.074 & 0.039 & \\
\hline \multicolumn{3}{|c|}{ The log. slope } & 0.86 & 1.37 & 1.48 & 1.48 & 0.76 & 1.30 & 1.49 & 1.54 \\
\hline
\end{tabular}

Figure 8 shows in a logarithmic scale the errors $e_{0}$ and $e_{1}$ versus $h$ for several values of $\mu$. Notice that $\log \left(e_{0}\right)$ and $\log \left(e_{1}\right)$ decrease quasi-linearly with respect of $\log h$. Average values of the slope of the curves $\log \left(e_{0}\right)$ and $\log \left(e_{1}\right)$ versus $\log h$ are presented in Table 1. One can notice on the one hand that these slopes increase when $\mu$ decreases, which confirms the predictions of Theorem 1. On the other hand, the values of the slope for the error $e_{0}$ are quite close to the values of the slope for the error $e_{1}$, even though $e_{0}$ is smaller than $e_{1}$. Namely, the weighted $L^{2}$ error $\left\|u-u_{h}\right\|_{\mathcal{H}_{-1}^{0}\left(\mathbb{R}_{+}^{3}\right)}$ does not converge faster than the error $\left\|u-u_{h}\right\|_{\mathcal{H}_{-1}^{1}\left(\mathbb{R}_{+}^{3}\right)}$. This fact confirms the lack of superconvergence of error in weighted $L^{2}:\left\|u-u_{h}\right\|_{\mathcal{H}_{-1}^{0}\left(\mathbb{R}_{+}^{3}\right)}$.

On the other hand, in order to show that the method is efficient for several kinds of behaviors at infinity, we display in Figure 9 the exact and the computed solutions for various values of $\epsilon$ (Ex. 2). Recall that $u \sim \rho^{-2(1+\epsilon)}$ when $|\boldsymbol{x}| \mapsto+\infty$ and that $u \in \mathcal{H}_{-1}^{1}$ iff $\epsilon<0.75$.

\subsubsection{Influence of the parameter $\gamma$}

The only effective constraint on the parameter $\gamma$ is the condition (57) which ensures the conformity. Since $\alpha=-1, n=3$ and $p=2$, this condition reduces here to

$$
\gamma>-\frac{1}{2}
$$

The condition (38) of Theorem 1 (which is more restrictive than (57)), indicates a manner of choosing $\gamma$, once one knows that the solution belong to the space $\mathcal{H}_{\beta}^{k+1}(\Omega)$ for some real $\beta$. In Table 2 is given the numerical error for several values of $\gamma$ when all the other parameters are fixed $(R=1, h=0.075(\mathrm{~N}=40), \mu=0.5$. Ex. 1 with $\epsilon=0$ ). One can observe that the error varies slowly with $\gamma$. However, the number of iterations necessary for the convergence of the CG algorithm (without preconditioning) depends strongly on $\gamma$. That means that $\gamma$ has a serious influence on the spectral properties of the matrix $A_{\gamma}$. Further, one can observe that there exists an optimal value of $\gamma \simeq 0.7$ for which both the error of approximation and the convergence of the CG algorithm are optimal.

\subsubsection{Influence of the size of the FEM region}

Despite the fact that the size of the FEM region $R$ is not meant to be large, it could be useful to study its influence on the error of approximation. Here, we made some computational tests with the parameters $\gamma$, $\mu$ and $D O F$ fixed and with $R$ varying from 1 to 14 . Example 2 is used in all these tests. Since the number of degrees of freedom is fixed, the discretization parameter $h$ increases linearly with $R$. Despite that fact, the global error of approximation decreases when $R$ is smaller than a value $R_{\text {opt }}$. It increases with $R$ when $R \geq R_{\text {opt }}$. Here $R_{\text {opt }}$ is an optimal radius for which the error is minimal. This radius depends slightly on the total number of degrees of freedom. In Figure 10 shows the relative error $e_{1}$ versus $R$ for $N=40(D O F=44361)$ and for $N=60(D O F=147741)(\mu=0.5$ and $\gamma=1$ in both the cases $)$. On the other hand, it is worth noting that the distribution of the error between the FEM region and the IFEM region depends also on the choice of 

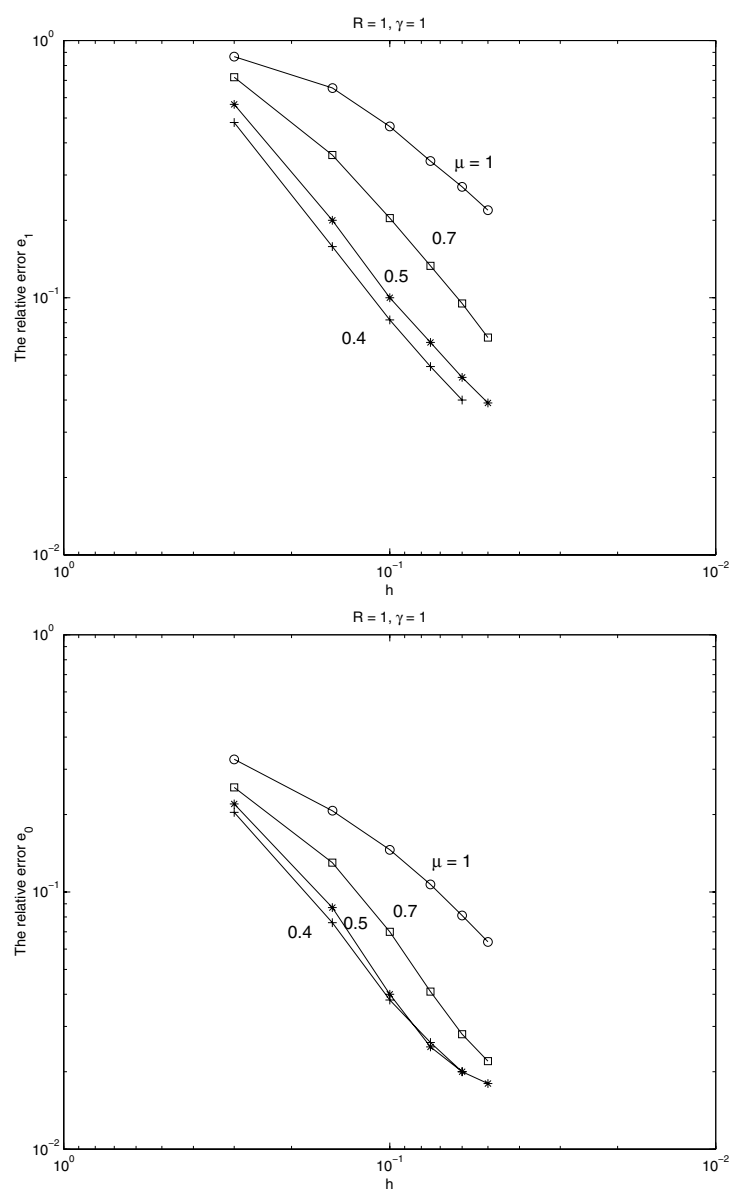

FiguRE 8. The relative errors $e_{0}$ and $e_{1}$ versus $h$ for several values of $\mu$ (in a logarithmic scale) $(R=1, \gamma=1$ and $\epsilon=0$. Example 1 with $\epsilon=0)$.

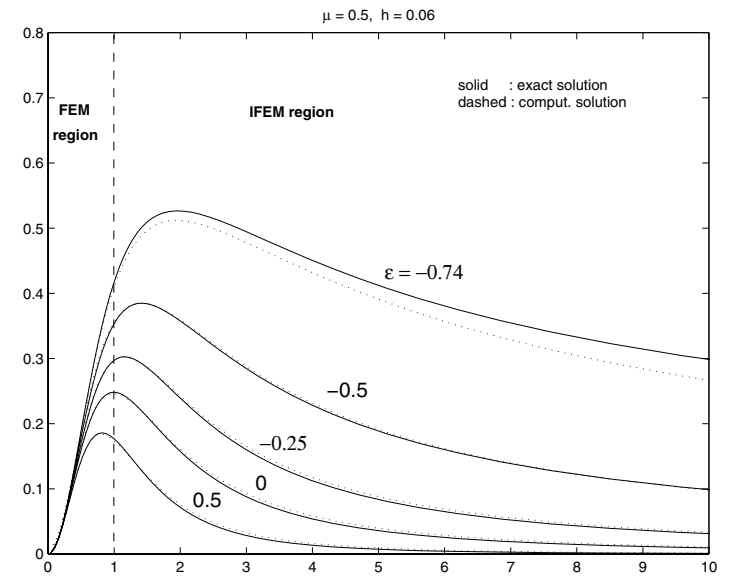

FIGURE 9. The exact and the approximate solutions for several behaviors at infinity. Here $R=1, \gamma=1+2 \epsilon, \mu=0.5$ and $N=50(h=0.06)$. 
TABLE 2. The relative errors and the number of iterations necessary for the convergence of CG algorithm versus the parameter $\gamma$.

\begin{tabular}{cccc}
\hline$\gamma$ & $e_{0}$ & $e_{1}$ & Number of iterations (CG)) \\
\hline-0.5 & 0.032 & 0.080 & 85489 \\
0 & 0.025 & 0.071 & 7689 \\
0.5 & 0.022 & 0.066 & 853 \\
0.7 & 0.023 & 0.066 & 464 \\
1. & 0.025 & 0.067 & 537 \\
1.5 & 0.032 & 0.072 & 1805 \\
2 & 0.046 & 0.081 & 15333 \\
2.5 & 0.071 & 0.095 & $>100000$ \\
\hline
\end{tabular}

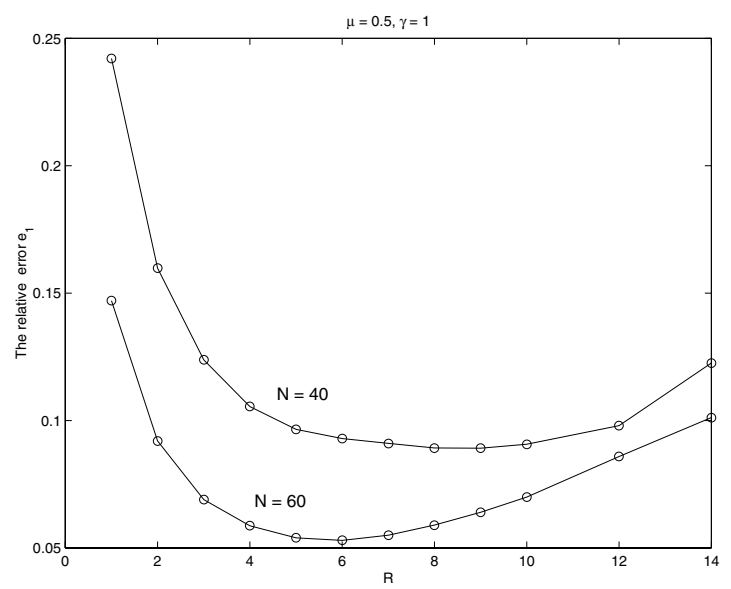

Figure 10. The relative error $e_{1}$ versus the shape parameter $R$ when $N=40$ and when $N=60$.

the parameter $R$. In Figure 11 the exact and the approximate solutions are plotted versus the radius $r$ when $x=y=0$ for three values of $R: R=2, R=4$ and $R=9$.

\section{A. Appendix. The proof of Proposition 1}

Proof. Let $u$ be a function in $\mathcal{H}_{\alpha}^{m, p}\left(\Omega_{\infty}\right)$. We set $\tilde{u}=\Lambda_{\gamma} u$. Then, for each $i \in\{1, . ., M\}$ we have the formula

$$
\int_{T_{i}} r(\boldsymbol{y})^{p \alpha}|u(\boldsymbol{y})|^{p} \mathrm{~d} \boldsymbol{y}=\int_{S_{i}} r(\boldsymbol{x})^{p(\gamma-\alpha)-2 n}|\tilde{u}(\boldsymbol{x})|^{p} \mathrm{~d} \boldsymbol{x},
$$

which follows from (22) and (28). Hence,

$$
\begin{aligned}
\int_{T_{i}}|\boldsymbol{y}|^{p \alpha}|u(\boldsymbol{y})|^{p} \mathrm{~d} \boldsymbol{y} & \simeq \int_{T_{i}} r(\boldsymbol{y})^{p \alpha}|u(\boldsymbol{y})|^{p} \mathrm{~d} \boldsymbol{y} \\
& \simeq \int_{S_{i}} r(\boldsymbol{x})^{p(\gamma-\alpha)-2 n}|\tilde{u}(\boldsymbol{x})|^{p} \mathrm{~d} \boldsymbol{x} \\
& \simeq \int_{S_{i}}|\boldsymbol{x}|^{p \delta}|\tilde{u}(\boldsymbol{x})|^{p} \mathrm{~d} \boldsymbol{x}
\end{aligned}
$$



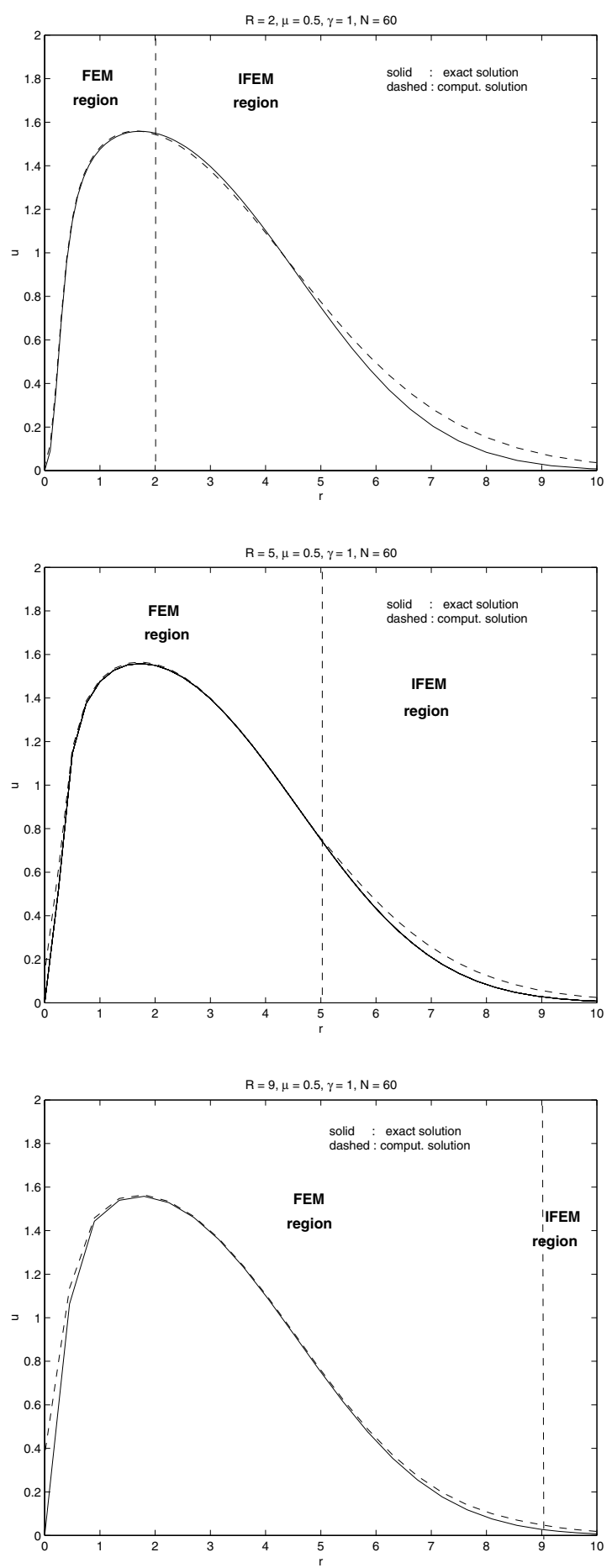

FiguRE 11. The exact and the approximate solution versus $r$ when $R=2$ (left), $R=5$ (center) and $R=9$ (right) (Ex. 2 with $N=60, \mu=0.5$ and $\gamma=1$ ). 
where $\delta=\gamma-\alpha-2 n / p$. We have also

$$
\begin{aligned}
\frac{\partial \tilde{u}}{\partial x_{k}}(\boldsymbol{x})= & -\gamma r(\boldsymbol{x})^{-\gamma-1} \frac{\boldsymbol{h}_{i} \cdot \boldsymbol{e}_{k}}{\left|\boldsymbol{h}_{i}\right|^{2}} u \circ \phi_{i}(\boldsymbol{x}) \\
& \left.+r(\boldsymbol{x})^{-\gamma}\left(\frac{\left|\boldsymbol{h}_{i}\right|^{4}}{\left(\boldsymbol{x} \cdot \boldsymbol{h}_{i}\right)^{2}}\left(\frac{\partial u}{\partial x_{k}}\right) \circ \phi_{i}(\boldsymbol{x})\right)-2 \frac{\left|\boldsymbol{h}_{i}\right|^{4}}{\left(\boldsymbol{x} \cdot \boldsymbol{h}_{i}\right)^{3}} \boldsymbol{h}_{i} \cdot \boldsymbol{e} k \boldsymbol{x} \cdot\left(\nabla u \circ \phi_{i}(\boldsymbol{x})\right)\right)
\end{aligned}
$$

for each $i \in\{1, \ldots, M\}$ and $\boldsymbol{x} \in S_{i}$. Thus,

$$
\left|\frac{\partial \tilde{u}}{\partial x_{k}}(\boldsymbol{x})\right| \lesssim|\boldsymbol{x}|^{-\gamma-1}|u(\boldsymbol{y})|+|\boldsymbol{x}|^{-\gamma-2}|\nabla \boldsymbol{u}(\boldsymbol{y})|,
$$

where $\boldsymbol{y}=\phi(\boldsymbol{x})$. It follows that

$$
\int_{S_{i}}|\boldsymbol{x}|^{p(\delta+1)}|\nabla \tilde{u}|^{p} \mathrm{~d} \boldsymbol{x} \lesssim \int_{T_{i}}\left(|\boldsymbol{y}|^{p \alpha}|u|^{p}+|\boldsymbol{y}|^{p(\alpha+1)}|\nabla u|^{p}\right) \mathrm{d} \boldsymbol{y} .
$$

We deduce that

Similarly, we prove that

$$
\|\tilde{u}\|_{V_{\delta}^{1, p}\left(S_{i}\right)} \lesssim\|u\|_{\mathcal{H}_{\alpha}^{1, p}\left(T_{i}\right)}
$$

$$
\|u\|_{\mathcal{H}_{\alpha}^{1, p}\left(T_{i}\right)} \lesssim\|\tilde{u}\|_{V_{\delta}^{1, p}\left(S_{i}\right)} .
$$

The proof can be extended by induction to the cases $m \geq 2$ :

$$
\|\tilde{u}\|_{V_{\delta}^{m, p}\left(S_{i}\right)} \simeq\|u\|_{\mathcal{H}_{\alpha}^{m, p}\left(T_{i}\right)}
$$

Now, suppose that $m=1$ and let us prove that $\tilde{u}$ belongs to $V_{\delta}^{1, p}\left(\Omega_{*}\right)$. This is mainly due to the continuity of $r(\boldsymbol{x})$. Indeed, let $\varphi \in \mathscr{D}\left(\Omega_{*}\right)$. We set

$$
\chi(\boldsymbol{x})=\varphi(\phi(\boldsymbol{x})) \text { for } \boldsymbol{x} \in \Omega_{\infty}
$$

Then, $\chi$ has compact support in $\Omega_{\infty}$ and its restriction to each sub-domain $T_{i}$ is $\mathscr{C}^{\infty}$. Note that

$$
\varphi(\boldsymbol{x})=\chi(\phi(\boldsymbol{x})) \text { for } \boldsymbol{x} \in \Omega_{*} .
$$

Applying the chain rule, we get

$$
\forall \boldsymbol{x} \in S_{i}, \nabla_{\boldsymbol{x}} \varphi=-\frac{2 r_{i}(\boldsymbol{y})}{\left|\boldsymbol{h}_{i}\right|^{2}}\left(\boldsymbol{y} . \nabla_{\boldsymbol{y}} \chi\right) \boldsymbol{h}_{i}+r_{i}(\boldsymbol{y})^{2} \frac{\partial \chi}{\partial y_{k}}(\boldsymbol{y}),
$$

where $\boldsymbol{y}=\phi(\boldsymbol{x}) \in T_{i}$. We get

$$
\begin{aligned}
\int_{S_{i}} \tilde{u}(\boldsymbol{x}) \frac{\partial \varphi}{\partial x_{k}} \mathrm{~d} \boldsymbol{x}= & \frac{1}{\left|\boldsymbol{h}_{i}\right|^{2}} \int_{T_{i}} r_{i}(\boldsymbol{y})^{\gamma+1-2 n} u(\boldsymbol{y})\left[-2\left(\boldsymbol{y} . \nabla_{\boldsymbol{y}} \chi\right)\left(\boldsymbol{h}_{i} \cdot \boldsymbol{e}_{k}\right)+\left|\boldsymbol{h}_{i}\right|^{2} r_{i}(\boldsymbol{y}) \frac{\partial \chi}{\partial y_{k}}\right] \mathrm{d} \boldsymbol{y} \\
= & \frac{2 \boldsymbol{h}_{i} \cdot \boldsymbol{e}_{k}}{\left|\boldsymbol{h}_{i}\right|^{2}} \int_{T_{i}} \operatorname{div}_{\boldsymbol{y}}\left[r_{i}(\boldsymbol{y})^{\gamma+1-2 n} u \boldsymbol{y}\right] \chi \mathrm{d} \boldsymbol{y}-2\left(\boldsymbol{h}_{i} \cdot \boldsymbol{e}_{k}\right) \int_{\partial T_{i}} r_{i}(\boldsymbol{y})^{\gamma+1} u(\boldsymbol{y}) \chi(\boldsymbol{y}) \boldsymbol{y} \cdot \boldsymbol{n}_{i} \mathrm{~d} \sigma \\
& -\int_{T_{i}} \frac{\partial}{\partial y_{k}}\left(r_{i}(\boldsymbol{y})^{\gamma+2} u(\boldsymbol{y})\right) \chi(\boldsymbol{y}) \mathrm{d} \boldsymbol{y}+2 \int_{\partial T_{i}} r_{i}(\boldsymbol{y})^{\gamma+2} u(\boldsymbol{y}) \chi(\boldsymbol{y}) \boldsymbol{n}_{i} . \boldsymbol{e}_{k} \mathrm{~d} \sigma
\end{aligned}
$$


where $\boldsymbol{n}_{i}$ denotes the exterior normal of $T_{i}$. Observe that $\chi=0$ on $\partial T_{i} \cap \partial S_{i}$ and that $\chi(\boldsymbol{y}) \boldsymbol{y} . \boldsymbol{n}_{i}=0$ on $\partial T_{i}$. Since the jump of $r(\boldsymbol{x})$ and $u(\boldsymbol{x})$ across the unbounded faces of $T_{i}$ is zero, we deduce easily that

$$
\int_{\Omega_{*}} \tilde{u}(\boldsymbol{x}) \frac{\partial \varphi}{\partial x_{k}} \mathrm{~d} \boldsymbol{x}=\int_{\Omega_{\infty}} v_{k}(\boldsymbol{y}) \chi(\boldsymbol{y}) \mathrm{d} \boldsymbol{y},
$$

where $v_{k}$ is given by

$$
v_{k}(\boldsymbol{y})=\frac{2 \boldsymbol{h}_{i} \cdot \boldsymbol{e}_{k}}{\left|\boldsymbol{h}_{i}\right|^{2}} \operatorname{div}_{\boldsymbol{y}}\left[r_{i}(\boldsymbol{y})^{\gamma+1-2 n} u \boldsymbol{y}\right]-\frac{\partial}{\partial y_{k}}\left(r_{i}(\boldsymbol{y})^{\gamma+2} u(\boldsymbol{y})\right), \text { for } \boldsymbol{y} \in T_{i} .
$$

Since $r(\boldsymbol{y}) \simeq|\boldsymbol{y}|$ in $\Omega_{\infty}$ and $\nabla u \in \mathcal{H}_{\alpha}^{1, p}\left(\Omega_{\infty}\right)$, we deduce that

$$
v_{k} \in \mathcal{H}_{\alpha+2 n-(\gamma+1)}^{0, p}\left(\Omega_{\infty}\right) .
$$

Hence, from (68) we get

$$
\begin{aligned}
\int_{\Omega_{*}} \tilde{u}(\boldsymbol{x}) \frac{\partial \varphi}{\partial x_{k}} \mathrm{~d} \boldsymbol{x} & =\int_{\Omega_{*}} r(\boldsymbol{x})^{-2 n}(v \circ \phi)(\boldsymbol{x}) \varphi(\boldsymbol{x}) \mathrm{d} \boldsymbol{x} \\
& =\int_{\Omega_{*}}\left(\Lambda_{2 n} v_{k}\right)(\boldsymbol{x}) \varphi(\boldsymbol{x}) \mathrm{d} \boldsymbol{x}
\end{aligned}
$$

As in the case $m=0$, we conclude that

$$
\frac{\partial \tilde{u}}{\partial x_{k}}=\Lambda_{2 n} v_{k} \in \mathcal{H}_{\delta+1}^{0, p}\left(\Omega_{\infty}\right)
$$

which ends the proof of Proposition 1.

\section{REFERENCES}

[1] R.A. Adams, Compact imbeddings of weighted Sobolev spaces on unbounded domains. J. Differential Equations 9 (1971) 325-334.

[2] F. Alliot and C. Amrouche, Problème de Stokes dans $\mathbb{R}^{n}$ et espaces de Sobolev avec poids. C. R. Acad. Sci. Paris Sér. I Math. 325 (1997) 1247-1252.

[3] C. Amrouche, V. Girault and J. Giroire, Weighted Sobolev spaces for Laplace's equation in $\mathbb{R}^{n}$. J. Math. Pures Appl. (9) $\mathbf{7 3}$ (1994) 579-606.

[4] C. Amrouche, V. Girault and J. Giroire, Dirichlet and Neumann exterior problems for the $n$-dimensional Laplace operator: an approach in weighted Sobolev spaces. J. Math. Pures Appl. (9) 76 (1997) 55-81.

[5] J. Bérenger, A perfectly matched layer for absoption of electromagnetics waves. J. Comput. Physics 114 (1994) 185-200.

[6] J. Bérenger, Perfectly matched layer for the fdtd solution of wave-structure interaction problems. IEEE Trans. Antennas Propagat. 44 (1996) 110-117.

[7] P. Bettess and O.C. Zienkiewicz, Diffraction and refraction of surface waves using finite and infinite elements. Internat. J. Numer. Methods Engrg. 11 (1977) 1271-1290.

[8] T.Z. Boulmezaoud, Vector potentials in the half-space of $\mathbb{R}^{3}$. C. R. Acad. Sci. Paris Sér. I Math. 332 (2001) 711-716.

[9] T.Z. Boulmezaoud, On the Stokes system and on the biharmonic equation in the half-space: an approach via weighted Sobolev spaces. Math. Methods Appl. Sci. 25 (2002) 373-398.

[10] T.Z. Boulmezaoud, On the Laplace operator and on the vector potential problems in the half-space: an approach using weighted spaces. Math. Methods Appl. Sci. 26 (2003) 633-669.

[11] D.S. Burnett, A three-dimensional acoustic infinite element based on a prolate spheroidal multipole expansion. J. Acoust. Soc. Amer. 96 (1994) 2798-2816.

[12] C. Canuto, S.I. Hariharan, L. Lustman, Spectral methods for exterior elliptic problems. Numer. Math. 46 (1985) 505-520.

[13] Y. Choquet-Bruhat and D. Christodoulou, Elliptic systems in $H_{s, \delta}$ spaces on manifolds which are Euclidean at infinity. Acta Math. 146 (1981) 129-150.

[14] Ph.-G. Ciarlet, The finite element method for elliptic problems. North-Holland Publishing Co., Amsterdam (1978). 
[15] D.L. Colton and R. Kress, Integral equation methods in scattering theory. Pure Appl. Math. John Wiley \& Sons Inc., New York (1983).

[16] L. Demkowicz and F. Ihlenburg, Analysis of a coupled finite-infinite element method for exterior Helmholtz problems. Numer. Math. 88 (2001) 43-73.

[17] J. Deny and J.L. Lions, Les espaces du type de Beppo Levi. Ann. Inst. Fourier, Grenoble 5 (1955) 305-370, (1953-54).

[18] K. Gerdes, A summary of infinite element formulations for exterior Helmholtz problems. Comput. Methods Appl. Mech. Engrg. 164 (1998) 95-105.

[19] K. Gerdes and L. Demkowicz, Solution of 3D-Laplace and Helmholtz equations in exterior domains using $h p$-infinite elements. Comput. Methods Appl. Mech. Engrg. 137 (1996) 239-273.

[20] V. Girault, The divergence, curl and Stokes operators in exterior domains of $\mathbb{R}^{n}$. In Recent developments in theoretical fluid mechanics (Paseky, 1992), Pitman Res. Notes Math. Ser. Longman Sci. Tech., Harlow 291 (1993) 34-77.

[21] V. Girault, The Stokes problem and vector potential operator in three-dimensional exterior domains: an approach in weighted Sobolev spaces. Differential Integral Equations 7 (1994) 535-570.

[22] J. Giroire, Étude de quelques problèmes aux limites extérieures et résolution par équations intégrales. Thèse de Doctorat d'Etat, Université Pierre et Marie Curie, Paris (1987).

[23] J. Giroire and J.-C. Nédélec, Numerical solution of an exterior Neumann problem using a double layer potential. Math. Comp. 32 (1978) 973-990.

[24] L. Halpern, A spectral method for the Stokes problem in three-dimensional unbounded domains. Math. Comp. 70 (2001) 1417-1436 (electronic).

[25] B. Hanouzet, Espaces de Sobolev avec poids application au problème de Dirichlet dans un demi espace. Rend. Sem. Mat. Univ. Padova 46 (1971) 227-272.

[26] G.H. Hardy, J.E. Littlewood and G. Pólya, Inequalities. Cambridge Mathematical Library. Cambridge University Press, Cambridge (1988).

[27] L. Hörmander and J.L. Lions, Sur la complétion par rapport à une intégrale de Dirichlet. Math. Scand. 4 (1956) $259-270$.

[28] F. Ihlenburg, Finite element analysis of acoustic scattering, volume 132 of Applied Mathematical Sciences. Springer-Verlag, New York (1998).

[29] V.A. Kondratev, Boundary value problems for elliptic equations in domains with conical or angular points. Trudy Moskov. Mat. Obvsvc. 16 (1967) 209-292.

[30] A. Kufner, Weighted Sobolev spaces. A Wiley-Interscience Publication. John Wiley \& Sons Inc., New York (1985).

[31] M. Laib and T.Z. Boulmezaoud, Some properties of weighted sobolev spaces in unbounded domains. In preparation.

[32] M.N. Le Roux, Méthode d'éléments finis pour la résolution numérique de problèmes extérieurs en dimension 2. RAIRO Anal. Numér. 11 (1977) 27-60.

[33] V.G. Maz'ya and B.A. Plamenevskii, Weighted spaces with inhomogeneous norms, and boundary value problems in domains with conical points, in Elliptische Differentialgleichungen (Meeting, Rostock, 1977). Wilhelm-Pieck-Univ. Rostock (1978) $161-190$.

[34] J.-C. Nédélec, Curved finite element methods for the solution of singular integral equations on surfaces in $\mathbb{R}^{3}$. Comput. Methods Appl. Mech. Engrg. 8 (1976) 61-80.

[35] J.-C. Nédélec. Résolution des Équations de Maxwell par Méthodes Intégrales. Cours de D.E.A. École Polytechnique, Paris (1998).

[36] V. Rokhlin, Solution of acoustic scattering problems by means of second kind integral equations. Wave Motion 5 (1983) $257-272$. 